\title{
eXtended Hybridizable Discontinuous Galerkin for incompressible flow problems with unfitted meshes and interfaces
}

\author{
Ceren Gürkan · Martin Kronbichler · Sonia \\ Fernández-Méndez
}

Received: date / Accepted: date

\begin{abstract}
The eXtended Hybridizable Discontinuous Galerkin (X-HDG) method is developed for the solution of Stokes problems with void or material interfaces. X-HDG is a novel method that combines the Hybridizable Discontinuous Galerkin (HDG) method with an eXtended Finite Element (X-FEM) strategy, resulting in a high-order, unfitted, superconvergent method, with an explicit definition of the interface geometry by means of a level-set function. For elements not cut by the interface, the standard HDG formulation is applied, whereas a modified weak form for the local problem is proposed for cut elements. Heaviside enrichment is considered on cut faces and in cut elements in the case of bimaterial problems. Two-dimensional numerical examples demonstrate that the applicability, accuracy, and superconvergence properties of HDG are inherited in X-HDG, with the freedom of computational meshes that do not fit the interfaces.
\end{abstract}

Keywords interface $\cdot$ bimaterial · void · unfitted · Hybridizable Discontinuous Galerkin (HDG) $\cdot$ high-order $\cdot$ level-set $\cdot$ X-FEM $\cdot$ X-HDG

\section{Introduction}

The eXtended Hybridizable Discontinuous Galerkin Method (X-HDG), initially proposed in $[15,14]$ for the Laplace equation, is further developed here for the solution of incompressible flow problems with material or void interfaces. X-HDG is a novel method combining the superconvergence and computational efficiency of the Hybridizable discontinuous Galerkin (HDG) method with the flexibility of an eXtended Finite Element (X-FEM) philosophy with respect to interface representation.

HDG $[5,8]$ outperforms other discontinuous Galerkin (DG) methods for the solution of steady problems or transient problems with implicit time integration: besides the accuracy

C. Gürkan and S. Fernández-Méndez

Laboratori de Càlcul Numèric (LaCàN), Universitat Politècnica de Catalunya (UPC), Barcelona, Spain.

E-mail: [ceren.gurkan, sonia.fernandez]@upc.edu

M. Kronbichler

Institute for Computational Mechanics, Technical University of Munich, Garching, Germany.

E-mail: kronbichler@1nm.mw.tum.de 
gain due to superconvergence, the hybridization process underlying HDG significantly reduces the number of degrees of freedom (DOFs) in the linear system, similarly to static condensation in the context of high-order continuous Galerkin (CG) finite elements. For incompressible flow problems $[6,24]$, the HDG condensed system involves only the trace of the velocity, i.e., the velocity at the mesh sides (faces in 3D), and the mean of the pressure in each element, i.e., only a scalar variable per element for any degree of approximation $k$, as unknowns. Thus, the number of DOFs for the velocity is slightly larger for HDG compared to CG, due to the duplication of the vertex nodes (and edge nodes in 3D), but HDG has fewer DOFs for the pressure. Moreover, the block structure of the sparsity pattern of the HDG matrices has been shown to be convenient for linear solvers, concluding that HDG is very competitive in front of CG, see $[20,13,28]$ for efficiency comparisons for heat and wave problems. A comparison of CPU time for the solution of 2D incompressible flow problems in [25] has found that HDG requires less CPU time for the direct linear solver than CG for the same level of accuracy. In addition, HDG is based on a mixed formulation that is stable even when all variables are approximated with polynomials of the same degree $k$. Thus, in the case of Stokes problems, HDG provides convergence of order $k+1$ in the $\mathcal{L}_{2}$ norm not only for the velocity but also for the derivative of the velocity and for the pressure and, consequently, for the stress. In addition, a simple element-by-element postprocess of the derivatives leads to a superconvergent approximation of the velocity, with convergence of order $k+2$ in the $\mathcal{L}_{2}$ norm. HDG has been successfully applied to the solution of Laplace and Stokes problems with interfaces in $[18,27]$, but with a mesh fitting to the interface.

Advanced techniques for interface problems, such as the eXtended Finite Element (XFEM) method [23] or standard discretizations with phase-field models [19], have proved their efficiency for the solution of multi-phase flow problems with non-fitted meshes. In particular, phase-field models for flow problems have been successfully solved with DG methods, see for instance [2,26], and the extension to HDG methods seems promising. The strongest points of phase-field models are their suitability to consider the physics and chemistry between the different phases in a natural way, getting rid of defining specific interface conditions, and the possibility of using standard continuous or discontinuous approximations for their solution. However, these models are based on a smeared representation of interfaces, whose resolution depends on the discretization size and, therefore, extensive refinement around interfaces is necessary in practice [1]. X-FEM methods, on the other hand, consider a sharp representation of the interface, which is usually implicitly represented by a level-set function, and impose proper interface conditions. This allows these methods to be accurate already for coarser meshes. The interface conditions are model dependent, see, for example, [11] and references therein, and [4,9] for high-order approximations.

The X-HDG method developed here combines the advantageous aspects of HDG and $\mathrm{X}$-FEM, leading to a high-order unfitted numerical method, with a level-set description of interfaces. X-HDG inherits the superconvergence properties of HDG, together with its reduced size of the linear system in the condensed variables, but with a mesh that does not need to fit to the interface. With X-HDG, the computational mesh just covers the domain, hence, the complexity of interface geometry does not add extra difficulty to the problem, and no remeshing is required for evolving interfaces.

Similarly to standard HDG, a new variable for the trace of the velocity at the mesh skeleton (i.e., the mesh sides) is introduced, and the Stokes equations are rewritten as a set of local problems, corresponding to Dirichlet Stokes problems in each element, and some global equations, imposing continuity of the tractions across element boundaries. For a noncut element, the solution of the local problem is exactly the same as for standard HDG. For elements cut by an interface, modified local problems are stated and modified weak forms 
are proposed, depending on the type of interface. In the case of bimaterial interfaces, the $\mathrm{X}$-HDG approximation space at cut faces and cut elements is enriched with a Heaviside function for all variables, in order to reproduce the discontinuities across the interface. For a bimaterial interface or a Neumann boundary, following the HDG philosophy, a trace variable is considered on the interface, similar to the one defined on the mesh skeleton, and continuity of the velocity and the tractions across the interface is imposed in weak form. However, this extra unknown can be isolated in the local problem using the interface conditions, and it does not appear in the final condensed system. The modified local solver for cut elements has the same structure as for standard elements: the solution at the element is expressed exclusively in terms of the trace variable on the element faces and, for bimaterial and Dirichlet interfaces, the mean of the pressure in the element.

To determine the trace variable on the element faces and the mean of the pressure, the global problem is stated as for standard HDG, but this time it is discretized with an enriched approximation in the case of cut faces. After condensation of the elemental variables, similarly to standard HDG, the X-HDG linear system of equations involves only the trace of the velocity on the mesh skeleton, and the elemental scalar for the mean of the pressure in standard elements and in elements cut by Dirichlet or bimaterial interfaces. Thus, the XHDG system preserves the reduced number of DOFs, with a suitable block structure, but with more DOFs (usually twice as many) for enriched faces.

The X-HDG method can also be interpreted as an immersed boundary method (IB), see [22] for an overview, or a method with unfitted mesh [16] with overlapping for bimaterial problems, using the HDG natural strategies to impose boundary conditions or interface continuity conditions on boundaries or interfaces.

Section 2 presents the X-HDG method for problems with unfitted boundary descriptions of Dirichlet or Neumann kind, i.e., a problem with voids behind the interface. The X-HDG method for bimaterial problems is presented in Section 3. In this case, the mesh is assumed to fit to the domain boundary, but not to the bimaterial interface. For the sake of simplicity, void and bimaterial interfaces are presented separately, but the extension of the formulation to problems with both material and void interfaces, or problems with other boundary conditions on the interface, is straightforward, following the ideas in this manuscript. Finally, the applicability, the accuracy and the superconvergence properties of X-HDG are demonstrated with numerical examples in Section 5. The proposed X-HDG formulation is applicable also to $3 \mathrm{D}$ problems. The main difficulty in $3 \mathrm{D}$ computations is the implementation of the modification of the numerical integration for cut elements and the numerical integration on the surface corresponding to the interface, which can be done following the proposals in [10] in the context of X-FEM.

\section{X-HDG formulation for void problems}

Let $\Omega \subset \mathbb{R}^{d}$ be a bounded domain with interior boundaries (also referred to as interfaces), that may be of Dirichlet $\mathcal{I}^{D}$ or Neumann $\mathcal{I}^{N}$ type, and an exterior boundary $\partial \Omega^{\text {ext }}:=$ $\partial \Omega \backslash\left(\mathcal{I}^{D} \cup \mathcal{I}^{N}\right)$. The following Stokes problem is considered,

$$
\begin{aligned}
-\boldsymbol{\nabla} \cdot(\nu \boldsymbol{\nabla u})+\boldsymbol{\nabla} p=\boldsymbol{f} & \text { in } \Omega \\
\boldsymbol{\nabla} \cdot \boldsymbol{u}=\mathbf{0} & \text { in } \Omega \\
(-\nu \boldsymbol{\nabla u}+p \mathbf{I}) \cdot \boldsymbol{n}=\boldsymbol{g} & \text { on } \mathcal{I}^{N} \\
\boldsymbol{u}=\boldsymbol{u}_{D} & \text { on } \mathcal{I}^{D} \\
\boldsymbol{u}=\boldsymbol{u}_{D} & \text { on } \partial \Omega^{\mathrm{ext}}
\end{aligned}
$$



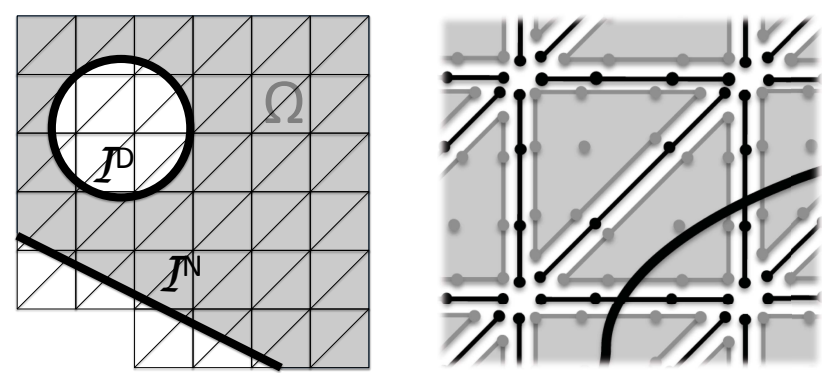

Fig. 1 Example of domain with two interfaces (left). The mesh covers the domain $\Omega$ (in gray) and fits the exterior boundary $\partial \Omega^{\text {ext }}$. Neumann and Dirichlet boundaries correspond to interfaces $\mathcal{I}^{D}$ and $\mathcal{I}^{N}$, that may cross some elements. Example of HDG computational mesh (right): representation of the nodes in the elements and in the faces (mesh skeleton) for degree $k=3$.

where $\boldsymbol{u}$ is the velocity, $p$ is the pressure, $\nu$ is the material viscosity, $\mathbf{I}$ is the second-order identity tensor, $\boldsymbol{f}$ is an external force, $\boldsymbol{u}_{D}$ are prescribed values on the Dirichlet boundaries $\mathcal{I}^{D}$ and $\partial \Omega^{\text {ext }}$, and $\boldsymbol{g}$ is a prescribed traction on the interior Neumann boundary $\mathcal{I}^{N}$. On the exterior boundary $\partial \Omega^{\text {ext }}$, Dirichlet boundary conditions are considered; however, other boundary conditions do not add any difficulty, since they are implemented as in standard HDG. The treatment of mixed boundary conditions on interfaces can also be deduced easily following the ideas in this section.

The domain $\Omega$ is now assumed to be covered by a finite element mesh with $\mathrm{n}_{\mathrm{el}}$ elements $\left\{K_{i}\right\}_{i=1}^{\mathrm{n}_{\mathrm{el}}}$, such that

$$
\bar{\Omega} \subset \bigcup_{i=1}^{\mathrm{n}_{\mathrm{e} 1}} \bar{K}_{i}, \quad K_{i} \cap K_{j}=\emptyset \text { for } i \neq j, \quad \partial \Omega^{\mathrm{ext}} \subset \partial\left[\bigcup_{i=1}^{\mathrm{n}_{\mathrm{e} 1}} \bar{K}_{i}\right]
$$

Note that the mesh fits the exterior boundary $\partial \Omega^{\text {ext }}$, but some elements may be cut by the interior boundaries $\mathcal{I}^{D}$ and $\mathcal{I}^{N}$, see Figure 1 . The union of all $\mathrm{n}_{\mathrm{fc}}$ faces $\Gamma_{f}$ (sides for 2D) intersecting the domain $\Omega$ is denoted as $\Gamma$, that is,

$$
\Gamma=\bigcup_{i=1}^{\mathrm{n}_{\mathrm{el}}}\left[\partial K_{i} \cap \bar{\Omega}\right]=\bigcup_{f=1}^{\mathrm{n}_{\mathrm{fc}}}\left[\Gamma_{f} \cap \bar{\Omega}\right] .
$$

Elements are classified in four groups: elements cut by the Dirichlet interface, $\mathcal{E}^{D}$, elements cut by the Neumann interface, $\mathcal{E}^{N}$, stardard elements, $\mathcal{E}^{S}$, and elements in the void. That is,

$$
\begin{aligned}
& \mathcal{E}^{D}=\left\{i \in \mathcal{E}: K_{i} \cap \mathcal{I}^{D} \neq \emptyset\right\}, \\
& \mathcal{E}^{N}=\left\{i \in \mathcal{E}: K_{i} \cap \mathcal{I}^{N} \neq \emptyset\right\}, \\
& \mathcal{E}^{S}=\left\{i \in \mathcal{E}: K_{i} \subset \Omega\right\},
\end{aligned}
$$

with $\mathcal{E}=\left\{1,2, \ldots, \mathrm{n}_{\mathrm{e}}\right\}$. Elements cut by two interfaces are not considered here for simplicity of explanations, but can be treated with similar procedures.

As in other X-FEM techniques, an interface may cut an element into two portions with very different size, leading to ill-conditioning problems that may drastically affect to the accuracy of the solution. In the numerical examples in this work, in the presence of bad-cut situations, the mesh is slightly modified according to [14]. 
Following the HDG rationale, problem (1) is split in a set of local problems, for each one of the elements, and some global equations defined on element faces. The local problems for the X-HDG formulation are

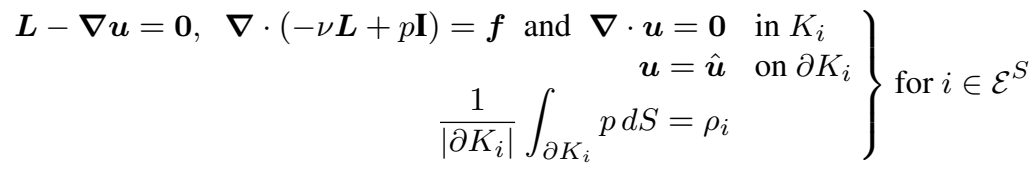

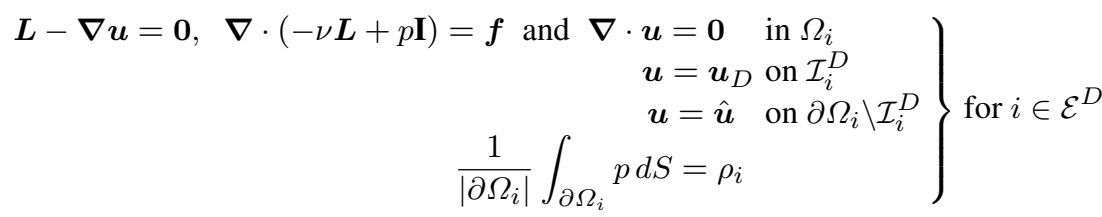

$$
\begin{aligned}
& \left.\begin{array}{rlrl}
\boldsymbol{L}-\boldsymbol{\nabla u}=\mathbf{0}, \boldsymbol{\nabla} \cdot(-\nu \boldsymbol{L}+p \mathbf{I})=\boldsymbol{f} \text { and } \boldsymbol{\nabla} \cdot \boldsymbol{u} & =\mathbf{0} & & \text { in } \Omega_{i} \\
(-\nu \boldsymbol{\nabla} \boldsymbol{u}+p \mathbf{I}) \cdot \boldsymbol{n} & =\boldsymbol{g} & & \text { on } \mathcal{I}_{i}^{N} \\
\boldsymbol{u} & =\hat{\boldsymbol{u}} & & \text { on } \partial \Omega_{i} \backslash \mathcal{I}_{i}^{N}
\end{array}\right\} \text { for } i \in \mathcal{E}^{N}
\end{aligned}
$$

with the following definitions for cut elements

$$
\Omega_{i}:=K_{i} \cap \Omega, \quad \mathcal{I}_{i}^{D}:=\mathcal{I}^{D} \cap K_{i}, \quad \mathcal{I}_{i}^{N}:=\mathcal{I}^{N} \cap K_{i} .
$$

As in standard HDG, the variable $\boldsymbol{L}$ is the gradient of $\boldsymbol{u}$, allowing the splitting of the PDE in two first order PDEs, $\hat{\boldsymbol{u}}$ is the trace of $\boldsymbol{u}$ at the mesh faces $\Gamma$, and $\rho_{i}$ is the mean of the pressure at the boundary of the element, which is only a scalar for each element. The new variables, $\hat{\boldsymbol{u}}$ and $\left\{\rho_{i}\right\}_{i \in \mathcal{E}^{S} \cup \mathcal{E}^{D}}$, are assumed to be data in the local problems.

The local problem (2a) corresponds to the Stokes problem with Dirichlet boundary conditions, $\boldsymbol{u}=\hat{\boldsymbol{u}}$, for an uncut element. Note that the Stokes problem with only Dirichlet data does not have a unique solution, and the last equation in (2a) closes the problem, setting the mean of the pressure on the element boundary to $\rho_{i}$. The local problem for an element cut by the Dirichlet interface (2b) is again a pure Dirichlet problem, with boundary conditions on the interface $\mathcal{I}_{i}^{D}$ and on the faces $\partial \Omega_{i} \backslash \mathcal{I}_{i}^{D}$, and the mean of the pressure is set to $\rho_{i}$ to ensure a unique solution. Finally, the local problem for an element cut by the Neumann interface (2c) corresponds to the Stokes problem in $\Omega_{i}$ with Neumann boundary conditions on the interface $\mathcal{I}_{i}^{N}$ and Dirichlet boundary conditions on the element faces, $\partial \Omega_{i} \backslash \mathcal{I}_{i}^{N}$. Note that, in the last case, the problem is not a pure Dirichlet problem and, therefore, the mean of the pressure is not set to any constant.

The local problems (2) can be solved element-by-element to determine $\boldsymbol{u}, \boldsymbol{L}$ and $p$, given $\hat{\boldsymbol{u}}$ and $\rho_{i}$. Thus, now the problem reduces to determine $\hat{\boldsymbol{u}}$ and $\left\{\rho_{i}\right\}_{i \in \mathcal{E}^{D} \cup \mathcal{E}^{S}}$ with the global equations

$$
\begin{gathered}
\llbracket(-\nu \boldsymbol{L}+p \mathbf{I}) \cdot \boldsymbol{n} \rrbracket=0 \quad \text { on } \Gamma \backslash \partial \Omega^{\mathrm{ext}}, \\
\int_{\partial K_{i} \cap \bar{\Omega}} \hat{\boldsymbol{u}} \cdot \boldsymbol{n} d S=\left\{\begin{array}{cc}
-\int_{\mathcal{I}_{i}^{D}} \boldsymbol{u}_{D} \cdot \boldsymbol{n} d S & \text { for } i \in \mathcal{E}^{D} \\
0 & \text { for } i \in \mathcal{E}^{S}
\end{array}\right. \\
\hat{\boldsymbol{u}}=\boldsymbol{u}_{D} \text { on } \partial \Omega^{\mathrm{ext}},
\end{gathered}
$$

where $\llbracket \cdot \rrbracket$ denotes the jump operator. On a face shared by two elements, $K_{L}$ and $K_{R}$, the jump is defined as

$$
\llbracket \boldsymbol{w} \cdot \boldsymbol{n} \rrbracket:=\boldsymbol{w}_{L} \cdot \boldsymbol{n}_{L}+\boldsymbol{w}_{R} \cdot \boldsymbol{n}_{R}=\left(\boldsymbol{w}_{L}-\boldsymbol{w}_{R}\right) \cdot \boldsymbol{n}_{L},
$$


where $\boldsymbol{w}$ may be discontinuous across the face, and the subcript denotes the element where the function, and the outward exterior normal, is evaluated.

Equation (3a) is the conservativity condition, weakly imposing continuity of the traction across element faces. Equation ( $3 b$ ) imposes the incompressibility condition on the boundary of the elements for $i \in \mathcal{E}^{S} \cup \mathcal{E}^{D}$, ensuring well-posedness of the Dirichlet local problems (2a) and (2b).

If the problem is a pure Dirichlet problem, i.e. $\mathcal{I}^{N}=\emptyset$, the solution of (1) is determined up to a constant for the pressure. In this case, an additional constraint for the pressure must be imposed at the global level, for instance, setting the mean of the pressure on the boundary of the first element to 0 , that is $\rho_{1}=0$, or to a given constant.

Discretization spaces,

$$
\begin{aligned}
& \mathcal{V}^{h}:=\left\{v \in \mathcal{L}_{2}(\Omega):\left.v\right|_{K_{i} \cap \Omega} \in \mathcal{P}_{k}\left(K_{i} \cap \Omega\right) \text { for } i \in \mathcal{E}^{S} \cup \mathcal{E}^{D} \cup \mathcal{E}^{N}\right\} \\
& \Lambda^{h}:=\left\{\hat{v} \in \mathcal{L}_{2}(\Gamma):\left.\hat{v}\right|_{\Gamma_{f} \cap \bar{\Omega}} \in \mathcal{P}_{k}\left(\Gamma_{f} \cap \bar{\Omega}\right) \text { for } f=1, \ldots, \mathrm{n}_{\mathrm{f}_{\mathrm{C}}}\right\},
\end{aligned}
$$

are considered for elemental variables $\boldsymbol{u}, \boldsymbol{L}$ and $p$, and for the trace variable $\hat{\boldsymbol{u}}$, respectively, where $\mathcal{P}_{k}$ denotes the space of polynomials of degree less or equal to $k$. The discretization of the local problems (2) and the global equations (3) leads to the complete X-HDG formulation detailed next.

In next sections the space $\mathcal{V}^{h}$ does not appear explicitly. It is replaced by its restriction to the element, that is $\mathcal{P}_{k}\left(K_{i}\right)$ or $\mathcal{P}_{k}\left(K_{i} \cap \Omega\right)$ for every element $K_{i}$, to highlight that local problems (2) are stated and solved element-by-element in a decoupled way, with elemental variables in the space $\mathcal{P}_{k}$ for each element. In all weak forms, the space for trace variables will appear as $\Lambda^{h}$, to highlight that trace variables are global variables linked in the whole mesh through the global equations (3).

\subsection{X-HDG discretization for void problems: local problems}

The X-HDG local problem for a standard element $K_{i}, i \in \mathcal{E}^{S}$, is the standard HDG local problem corresponding to the discretization of (2a): given $\hat{\boldsymbol{u}}_{h} \in\left[\Lambda^{h}\right]^{d}$ and $\rho_{i} \in \mathbb{R}$, find $\boldsymbol{u}_{h} \in\left[\mathcal{P}_{k}\left(K_{i}\right)\right]^{d}, \boldsymbol{L}_{h} \in\left[\mathcal{P}_{k}\left(K_{i}\right)\right]^{d \times d}$ and $p_{h} \in \mathcal{P}_{k}\left(K_{i}\right)$ such that

$$
\begin{gathered}
\int_{K_{i}}\left(-\boldsymbol{\nabla} \cdot\left(\nu \boldsymbol{L}_{h}\right)+\nabla p_{h}\right) \cdot \boldsymbol{v} d V+\int_{\partial K_{i}} \tau \nu\left(\boldsymbol{u}_{h}-\hat{\boldsymbol{u}}_{h}\right) \cdot \boldsymbol{v} d S=\int_{K_{i}} \boldsymbol{f} \cdot \boldsymbol{v} d V \\
\int_{K_{i}} \boldsymbol{L}_{h}: \boldsymbol{Q} d V+\int_{K_{i}}(\boldsymbol{\nabla} \cdot \boldsymbol{Q}) \cdot \boldsymbol{u}_{h} d V-\int_{\partial K_{i}}(\boldsymbol{Q} \cdot \boldsymbol{n}) \cdot \hat{\boldsymbol{u}}_{h} d S=0 \\
\int_{K_{i}} \boldsymbol{u}_{h} \cdot \boldsymbol{\nabla} q d V-\int_{\partial K_{i}}\left(\hat{\boldsymbol{u}}_{h} \cdot \boldsymbol{n}\right) q d S=0 \\
\frac{1}{\left|\partial K_{i}\right|} \int_{\partial K_{i}} p_{h} d S=\rho_{i},
\end{gathered}
$$

for all $\boldsymbol{v} \in\left[\mathcal{P}_{k}\left(K_{i}\right)\right]^{d}, \boldsymbol{Q} \in\left[\mathcal{P}_{k}\left(K_{i}\right)\right]^{d \times d}$ and $q \in \mathcal{P}_{k}\left(K_{i}\right)$. The first equation in (4) can be derived from the first equation in (2a) by applying integration by parts, replacing the velocity gradient by the numerical velocity gradient,

$$
\widehat{\boldsymbol{L}}:=\boldsymbol{L}+\tau(\hat{\boldsymbol{u}}-\boldsymbol{u}) \otimes \boldsymbol{n},
$$

and undoing the integration by parts. The parameter $\tau$ is an stabilization parameter, here taken as $\tau=1$, see [7,12] for details. The second and third equations are obtained from the 
weak form of the second and third equations in (2a) by simply applying integration by parts and replacing the boundary condition $\boldsymbol{u}=\hat{\boldsymbol{u}}$ on the element boundary.

The X-HDG local problem for an element cut by the Dirichlet interface, $K_{i}$ with $i \in$ $\mathcal{E}^{D}$, corresponds to the discretization of (2b): given $\hat{\boldsymbol{u}}_{h} \in\left[\Lambda^{h}\right]^{d}$ and $\rho_{i} \in \mathbb{R}$, find $\boldsymbol{u}_{h} \in$ $\left[\mathcal{P}_{k}\left(\Omega_{i}\right)\right]^{d}, \boldsymbol{L}_{h} \in\left[\mathcal{P}_{k}\left(\Omega_{i}\right)\right]^{d \times d}, p_{h} \in \mathcal{P}_{k}\left(\Omega_{i}\right)$ such that

$$
\begin{gathered}
\int_{\Omega_{i}}\left(-\boldsymbol{\nabla} \cdot\left(\nu \boldsymbol{L}_{h}\right)+\nabla p_{h}\right) \cdot \boldsymbol{v} d V+\int_{\partial \Omega_{i} \backslash \mathcal{I}_{i}^{D}} \nu \tau\left(\boldsymbol{u}_{h}-\hat{\boldsymbol{u}}_{h}\right) \cdot \boldsymbol{v} d S \\
+\int_{\mathcal{I}_{i}^{D}} \nu \tau \boldsymbol{u}_{h} \cdot \boldsymbol{v} d S=\int_{\Omega_{i}} \boldsymbol{f} \cdot \boldsymbol{v} d V+\int_{\mathcal{I}_{i}^{D}} \nu \tau \boldsymbol{u}_{D} \cdot \boldsymbol{v} d S \\
\int_{\Omega_{i}} \boldsymbol{L}_{h}: \boldsymbol{Q} d V+\int_{\Omega_{i}}(\boldsymbol{\nabla} \cdot \boldsymbol{Q}) \cdot \boldsymbol{u}_{h} d V \\
-\int_{\partial \Omega_{i} \backslash \mathcal{I}_{i}^{D}}(\boldsymbol{Q} \cdot \boldsymbol{n}) \cdot \hat{\boldsymbol{u}}_{h} d S=\int_{\mathcal{I}_{i}^{D}}(\boldsymbol{Q} \cdot \boldsymbol{n}) \cdot \boldsymbol{u}_{D} d S \\
\int_{\Omega_{i}} \boldsymbol{u}_{h} \cdot \boldsymbol{\nabla} q d V-\int_{\partial \Omega_{i} \backslash \mathcal{I}_{i}^{D}}\left(\hat{\boldsymbol{u}}_{h} \cdot \boldsymbol{n}\right) q d S=\int_{\mathcal{I}_{i}^{D}}\left(\boldsymbol{u}_{D} \cdot \boldsymbol{n}\right) q d S \\
\frac{1}{\left|\partial \Omega_{i}\right|} \int_{\partial \Omega_{i}} p_{h} d S=\rho_{i}
\end{gathered}
$$

for all $\boldsymbol{v} \in\left[\mathcal{P}_{k}\left(\Omega_{i}\right)\right]^{d}, \boldsymbol{Q} \in\left[\mathcal{P}_{k}\left(\Omega_{i}\right)\right]^{d \times d}$ and $q \in \mathcal{P}_{k}\left(\Omega_{i}\right)$. This local problem is exactly the same as the local problem for standard elements (4), just integrating in the portion of the element that is in the domain, $\Omega_{i}$, splitting the integrals on the Dirichlet boundary in integrals on the faces and integrals on the Dirichlet interface, and replacing the Dirichlet boundary data, $\boldsymbol{u}=\boldsymbol{u}_{D}$, for the second ones.

The X-HDG local problem for an element cut by the Neumann interface, $K_{i}$ with $i \in$ $\mathcal{E}^{N}$, corresponds to the discretization of (2c): given $\hat{\boldsymbol{u}}_{h} \in\left[\Lambda^{h}\right]^{d}$, find $\boldsymbol{u}_{h} \in\left[\mathcal{P}_{k}\left(\Omega_{i}\right)\right]^{d}$, $\boldsymbol{L}_{h} \in\left[\mathcal{P}_{k}\left(\Omega_{i}\right)\right]^{d \times d}, p_{h} \in \mathcal{P}_{k}\left(\Omega_{i}\right), \widetilde{\boldsymbol{u}}_{h}^{i} \in\left[\mathcal{P}_{k}\left(\mathcal{I}_{i}^{N}\right)\right]^{d}$ such that

$$
\begin{array}{r}
\int_{\Omega_{i}}\left(-\boldsymbol{\nabla} \cdot\left(\nu \boldsymbol{L}_{h}\right)+\nabla p_{h}\right) \cdot \boldsymbol{v} d V+\int_{\partial \Omega_{i} \backslash \mathcal{I}_{i}^{N}} \nu \tau\left(\boldsymbol{u}_{h}-\hat{\boldsymbol{u}}_{h}\right) \cdot \boldsymbol{v} d S \\
+\int_{\mathcal{I}_{i}^{N}} \nu \tau\left(\boldsymbol{u}_{h}-\widetilde{\boldsymbol{u}}_{h}^{i}\right) \cdot \boldsymbol{v} d S=\int_{\Omega_{i}} \boldsymbol{f} \cdot \boldsymbol{v} d V \\
\int_{\Omega_{i}} \boldsymbol{L}_{h}: \boldsymbol{Q} d V+\int_{\Omega_{i}}(\boldsymbol{\nabla} \cdot \boldsymbol{Q}) \cdot \boldsymbol{u}_{h} d V \\
-\int_{\partial \Omega_{i} \backslash \mathcal{I}_{i}^{N}}(\boldsymbol{Q} \cdot \boldsymbol{n}) \cdot \hat{\boldsymbol{u}}_{h} d S-\int_{\mathcal{I}_{i}^{N}}(\boldsymbol{Q} \cdot \boldsymbol{n}) \cdot \widetilde{\boldsymbol{u}}_{h}^{i} d S=0 \\
\int_{\Omega_{i}} \boldsymbol{u}_{h} \cdot \boldsymbol{\nabla} q d V-\int_{\partial \Omega_{i} \backslash \mathcal{I}_{i}^{N}}\left(\hat{\boldsymbol{u}}_{h} \cdot \boldsymbol{n}\right) q d S-\int_{\mathcal{I}_{i}^{N}}\left(\widetilde{\boldsymbol{u}}_{h}^{i} \cdot \boldsymbol{n}\right) q d S=0 \\
\int_{\mathcal{I}_{i}^{N}}\left(-\nu \boldsymbol{L}_{h} \cdot \boldsymbol{n}+p_{h} \boldsymbol{n}\right) \cdot \widetilde{\boldsymbol{v}} d S+\int_{\mathcal{I}_{i}^{N}} \tau \nu\left(\boldsymbol{u}_{h}-\widetilde{\boldsymbol{u}}_{h}^{i}\right) \cdot \widetilde{\boldsymbol{v}} d S=\int_{\mathcal{I}_{i}^{N}} \mathbf{g} \cdot \widetilde{\boldsymbol{v}} d S
\end{array}
$$

for all $\boldsymbol{v} \in\left[\mathcal{P}_{k}\left(\Omega_{i}\right)\right]^{d}, \boldsymbol{Q} \in\left[\mathcal{P}_{k}\left(\Omega_{i}\right)\right]^{d \times d}, q \in \mathcal{P}_{k}\left(\Omega_{i}\right)$ and $\widetilde{\boldsymbol{v}} \in\left[\mathcal{P}_{k}\left(\mathcal{I}_{i}^{N}\right)\right]^{d}$. Comparing with the previous local problems, the local problem for an element cut by the Neumann boundary does not require setting the constant for the pressure. Moreover, now the trace of the velocity on the interface is not a data, rather a new unknown $\widetilde{\boldsymbol{u}}_{h}^{i}$ in the local problem, following the HDG philosophy. This new unknown is local to an element and it is determined using the discretization of the Neumann boundary condition on the interface, i.e., the last equation in (7), which corresponds to the discretization of (3a) with the numerical velocity gradient

$$
\widetilde{\boldsymbol{L}}=\boldsymbol{L}+\tau\left(\widetilde{\boldsymbol{u}}^{i}-\boldsymbol{u}\right) \otimes \boldsymbol{n} \quad \text { on } \mathcal{I}_{i}^{N} .
$$



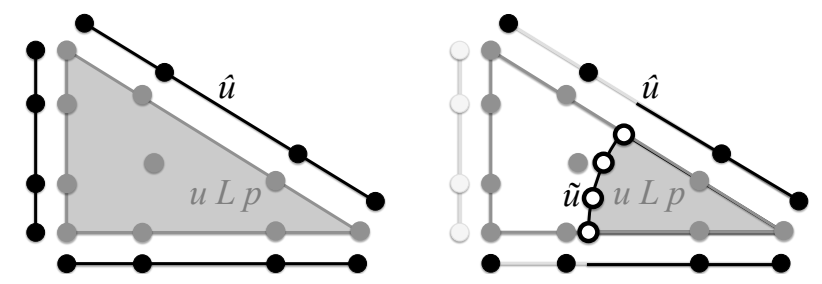

Fig. 2 Representation of the nodes for approximation for an standard element (left), and for an element cut by the interface (right), for an approximation of degree $k=3$. The nodal basis is defined in the whole element, and on the faces intersecting the domain, but numerical integration in done only in the gray area of the element and on the black portion of the faces associated with the domain $\Omega$. The $r+1$ white nodes on the interface, in this case with $r=k=3$, define the parametrization of degree $k$ approximating the interface in the element, in this case an isoparametric transformation from $(-1,1)$ to the curved segment.

The local problems in cut elements, (6) and (7), involve integrals in the portion of the element in the domain, $\Omega_{i}$, and on the interface, $\mathcal{I}_{i}$. Proper numerical quadrature rules to integrate in cut elements and interfaces are considered according to $[4,15]$. The interface $\mathcal{I}_{i}^{N}$ is approximated as a curved segment defined by an $r$-th degree polynomial parametrization, which is given by $r+1$ nodes on the interface, that is, the standard isoparametric transformation from $(-1,1)$ to a curved segment. A typical representation for $r=k=3$ is given in the right panel of Figure 2, see Section 4 for further details. The $r$-th degree parametrization is used to define numerical quadratures on the interface, and on the cropped element.

Although the approximation in a cut element, or on a cut face, is defined as a polynomial function in $\Omega_{i}=K_{i} \cap \Omega$, or on $\Gamma_{f} \cap \Omega$, respectively, standard nodal basis functions in the whole element $K_{i}$, or face $\Gamma_{f}$, are considered. That is, the reference element is the standard one but with a modified numerical quadrature rule to integrate only in the domain $\Omega$. In the example in Figure 2 right, polynomial nodal basis functions associated to all nodes (in gray) are considered in the element, and they are integrated only in the gray area. On faces intersecting the domain, nodal basis functions for all nodes (in black) are considered, and they are integrated only in the black portion of the face. Faces not intersecting the domain are disregarded in the integration.

The functional approximation space on the interface $\mathcal{P}_{k}\left(\mathcal{I}_{i}^{N}\right)$ is defined as the transformation of the one-dimensional $k$-th degree polynomial space from $(-1,1)$ to the curved segment approximating the interface $\mathcal{I}_{i}^{N}$. The standard nodal basis in $(-1,1)$ is mapped to the curved segment by means of the usual isoparametric transformation given by the coordinates of the $r+1$ nodes on $\mathcal{I}_{i}^{N}$.

The local problem for a standard element (4) or an element cut by the Dirichlet interface (6) leads to a system of equations of the form

$$
\left[\begin{array}{cccc}
\mathbf{A}_{u u}^{i} & \mathbf{A}_{u L}^{i} & \mathbf{A}_{u p}^{i} & \mathbf{0} \\
\mathbf{A}_{L u}^{i} & \mathbf{A}_{L L}^{i} & \mathbf{0} & \mathbf{0} \\
\mathbf{A}_{p u}^{i} & \mathbf{0} & \mathbf{0} & \mathbf{A}_{\rho p}^{i} T \\
\mathbf{0} & \mathbf{0} & \mathbf{A}_{\rho p}^{i} & \mathbf{0}
\end{array}\right]\left[\begin{array}{c}
\mathbf{u}^{i} \\
\mathbf{L}^{i} \\
\mathbf{p}^{i} \\
\lambda
\end{array}\right]=\left[\begin{array}{c}
\mathbf{f}_{u}^{i} \\
\mathbf{f}_{L}^{i} \\
\mathbf{f}_{p}^{i} \\
\mathbf{0}
\end{array}\right]+\left[\begin{array}{c}
\mathbf{0} \\
\mathbf{0} \\
\mathbf{0} \\
\mathbf{1}
\end{array}\right] \rho_{i}-\left[\begin{array}{c}
\mathbf{A}_{u \widehat{u}}^{i} \\
\mathbf{A}_{L \widehat{u}}^{i} \\
\mathbf{A}_{p \widehat{u}}^{i} \\
\mathbf{0}
\end{array}\right] \mathbf{\Lambda}^{i}
$$

with different expressions for the matrices and vectors, depending on the kind of element. In system (9) the constraint for the mean of the pressure, $\frac{1}{\left|\partial \Omega_{i}\right|} \int_{\partial \Omega_{i}} p d S=\rho_{i}$, is imposed with a Lagrange multiplier $\lambda$, the vectors $\mathbf{u}^{i}, \mathbf{L}^{i}$ and $\mathbf{p}^{i}$ are elemental vectors of nodal values, and $\boldsymbol{\Lambda}^{i}$ is a vector with the nodal values of the trace of the velocity $\hat{\boldsymbol{u}}^{f}$ for the faces of the 
element. This system can be solved for each element, leading to the so-called local solver in the element, that is, an explicit expression of the elemental variables $\mathbf{u}^{i}, \mathbf{L}^{i}$ and $\mathbf{p}^{i}$, in terms of the trace of the velocity at the faces $\Lambda^{i}$ and the mean of the pressure $\rho_{i}$,

$$
\mathbf{u}^{i}=\mathbf{S}_{u}^{i} \boldsymbol{\Lambda}^{i}+\mathbf{r}_{u}^{i} \rho_{i}+\mathbf{f}_{U}^{i}, \quad \boldsymbol{L}^{i}=\mathbf{S}_{L}^{i} \boldsymbol{\Lambda}^{i}+\mathbf{r}_{L}^{i} \rho_{i}+\mathbf{f}_{L}^{i}, \quad \mathbf{p}^{i}=\mathbf{S}_{p}^{i} \boldsymbol{\Lambda}^{i}+\mathbf{r}_{p}^{i} \rho_{i}+\mathbf{f}_{p}^{i},
$$

where the matrices $\mathbf{S}_{*}^{i}$ and the vectors $\mathbf{r}_{*}^{i}, \mathbf{f}_{*}^{i}$ depend on the matrices and vectors in (9).

In the case of an element cut by the Neumann interface (7), the system of equations is of the form

$$
\left[\begin{array}{cccc}
\mathbf{A}_{u u}^{i} & \mathbf{A}_{u L}^{i} & \mathbf{A}_{u p}^{i} & \mathbf{A}_{u \widetilde{u}}^{i} \\
\mathbf{A}_{L u}^{i} & \mathbf{A}_{L L}^{i} & \mathbf{0} & \mathbf{A}_{L \widetilde{u}}^{i} \\
\mathbf{A}_{p u}^{i} & \mathbf{0} & \mathbf{0} & \mathbf{A}_{p \widetilde{u}}^{i} \\
\mathbf{A}_{\widetilde{u} u}^{i} & \mathbf{A}_{\widetilde{u} L}^{i} & \mathbf{A}_{\widetilde{u} p}^{i} & \mathbf{A}_{\widetilde{u} \widetilde{u}}^{i}
\end{array}\right]\left[\begin{array}{c}
\mathbf{u}^{i} \\
\mathbf{L}^{i} \\
\mathbf{p}^{i} \\
\widetilde{\mathbf{u}}^{i}
\end{array}\right]=\left[\begin{array}{c}
\mathbf{f}_{u}^{i} \\
\mathbf{0} \\
\mathbf{0} \\
\mathbf{g}^{i}
\end{array}\right]-\left[\begin{array}{c}
\mathbf{A}_{u \widehat{u}}^{i} \\
\mathbf{A}_{L \widehat{u}}^{i} \\
\mathbf{A}_{p \widehat{u}}^{i} \\
\mathbf{0}
\end{array}\right] \mathbf{\Lambda}^{i}
$$

Thus, the local solver can be written as

$$
\mathbf{u}^{i}=\mathbf{S}_{u}^{i} \Lambda^{i}+\mathbf{f}_{U}^{i}, \quad \boldsymbol{L}^{i}=\mathbf{S}_{L}^{i} \boldsymbol{\Lambda}^{i}+\mathbf{f}_{L}^{i}, \quad \mathbf{p}^{i}=\mathbf{S}_{p}^{i} \boldsymbol{\Lambda}^{i}+\mathbf{f}_{p}^{i},
$$

expressing the elemental nodal values, $\mathbf{u}^{i}, \mathbf{L}^{i}$ and $\mathbf{p}^{i}$, in terms of the trace of the velocity at the faces $\boldsymbol{\Lambda}^{i}$. The trace on the interface $\widetilde{\mathbf{u}}^{i}$ is an elemental variable that could also be expressed in terms of the trace values in $\boldsymbol{\Lambda}^{i}$. However, its explicit expression is not used, because $\widetilde{\mathbf{u}}^{i}$ is just an auxiliary variable in the local problem, not present in the global equations.

\subsection{X-HDG discretization for void problems: global problem}

The X-HDG global problem corresponding to the discretization of (3) with the numerical velocity gradient (5) is: find $\hat{\boldsymbol{u}}_{h} \in\left[\Lambda^{h}\right]^{d}$ and $\rho^{i} \in \mathbb{R}$ for $i \in \mathcal{E}^{S} \cup \mathcal{E}^{D}$ satisfying

$$
\int_{\Gamma} \widehat{\boldsymbol{v}} \cdot \llbracket\left(-\nu \boldsymbol{L}_{h}+p_{h} \mathbf{I}\right) \cdot \boldsymbol{n} \rrbracket d S+2 \int_{\Gamma} \widehat{\boldsymbol{v}} \cdot\left(\left\{\nu \tau \boldsymbol{u}_{h}\right\}-\{\nu \tau\} \hat{\boldsymbol{u}}_{h}\right) d S=0
$$

for all $\widehat{\boldsymbol{v}} \in\left[\Lambda^{h}\right]^{d}$ such that $\widehat{\boldsymbol{v}}=0$ on $\partial \Omega^{\mathrm{ext}}$,

$$
\int_{\partial K_{i} \cap \bar{\Omega}} \hat{\boldsymbol{u}}_{h} \cdot \boldsymbol{n} d S=\left\{\begin{array}{cc}
-\int_{\mathcal{I}_{i}^{D}} \boldsymbol{u}_{D} \cdot \boldsymbol{n} d S & \text { for } i \in \mathcal{E}^{D} \\
0 & \text { for } i \in \mathcal{E}^{S}
\end{array}\right.
$$

and

$$
\hat{\boldsymbol{u}}_{h}=\mathbb{P}_{2}\left(\boldsymbol{u}_{D}\right) \quad \text { on } \partial \Omega^{\mathrm{ext}},
$$

where $\mathbb{P}_{2}$ denotes the $\mathcal{L}_{2}$ projection onto the discrete space on the exterior boundary, $\{\cdot\}$ denotes the mean operator, and $\boldsymbol{u}_{h}, \boldsymbol{L}_{h}$ and $p_{h}$ are solutions to the local problem for each element, i.e., the solution of (4), (6) or (7). The mean of a function $w$ on a face shared by two elements $K_{L}$ and $K_{R}$ is defined as

$$
\{w\}:=\frac{1}{2}\left(w_{L}+w_{R}\right)
$$


Equations (12) and (13) can be written in matrix form as

$$
\begin{aligned}
\mathbf{A}_{\widehat{u} u}^{f, L} \mathbf{u}^{L(f)}+ & \mathbf{A}_{\widehat{u} \boldsymbol{L}}^{f, L} \boldsymbol{L}^{L(f)}+\mathbf{A}_{\widehat{u} p}^{f, L} \mathbf{p}^{L(f)} \\
& +\mathbf{A}_{\widehat{u} u}^{f, R} \mathbf{u}^{R(f)}+\mathbf{A}_{\widehat{u} \boldsymbol{L}}^{f, R} \boldsymbol{L}^{R(f)}+\mathbf{A}_{\widehat{u} p}^{f, R} \mathbf{p}^{R(f)}+\mathbf{A}_{\widehat{u} \widehat{u}}^{f} \widehat{\mathbf{u}}^{f}=0
\end{aligned}
$$

for $f=1, \ldots, \mathrm{n}_{\mathrm{fC}}$, and

$$
\mathbf{A}_{\rho \widehat{u}} \mathbf{\Lambda}^{i}= \begin{cases}\mathbf{f}_{D}^{i} & \text { for } i \in \mathcal{E}^{D}, \\ 0 & \text { for } i \in \mathcal{E}^{S},\end{cases}
$$

where $R(f)$ and $L(f)$ denote the right and left elements sharing the face $f$. Replacing the local solvers - that is, equations (10) or (11) for the elements $K_{L(f)}$ and $K_{R(f)}$ - in (15) and (16), and applying the Dirichlet boundary condition (14), the global problem leads to a system of equations involving only the trace variable $\left\{\widehat{\mathbf{u}}^{f}\right\}_{f=1}^{n_{f c}}$ and the mean of the pressure

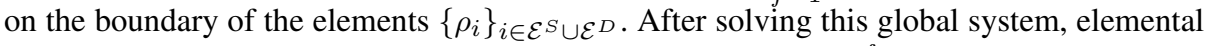
variables can be obtained by simply plugging in the solution, $\left\{\widehat{\mathbf{u}}^{f}\right\}_{f=1}^{\mathrm{n}_{\mathrm{f}_{\mathrm{C}}}}$ and $\left\{\rho_{i}\right\}_{i \in \mathcal{E} S \cup \mathcal{E}^{D}}$, in the local solver for each element.

From an implementation point of view, the trace vector $\Lambda^{i}$ can be defined to include the nodal values only for faces intersecting the domain. However, the simplest implementation assembles for all faces, even if they do not intersect the actual computational domain $\Omega$. The matrices assembled for faces not intersecting the domain are void matrices and, therefore, the corresponding rows and columns in the global system are void. Those rows and columns are removed from the system, reducing its size and rendering the system solvable with unique solution, after imposing boundary conditions.

\subsection{X-HDG discretization for void problems: postprocess for superconvergent solution}

Analogously to standard HDG, an element-by-element postprocessing provides a superconvergent solution. The procedure in a standard or cropped element, $\Omega_{i}=\Omega \cap K_{i}$, is: given $\boldsymbol{u}_{h} \in\left[\mathcal{P}_{k}\left(\Omega_{i}\right)\right]^{d}$ and $\boldsymbol{L}_{h} \in\left[\mathcal{P}_{k}\left(\Omega_{i}\right)\right]^{d \times d}$, find $\boldsymbol{u}_{h}^{*} \in\left[\mathcal{P}_{k+1}\left(\Omega_{i}\right)\right]^{d}$ such that

$$
\begin{gathered}
\int_{\Omega_{i}} \boldsymbol{\nabla} \boldsymbol{u}_{h}^{*}: \boldsymbol{\nabla v} d V=\int_{K_{i}} \boldsymbol{L}_{h}: \nabla \boldsymbol{v} d V \quad \forall \boldsymbol{v} \in\left[\mathcal{P}_{k+1}\left(\Omega_{i}\right)\right]^{d}, \\
\int_{\Omega_{i}} \boldsymbol{u}_{h}^{*} d V=\int_{\Omega_{i}} \boldsymbol{u}_{h} d V .
\end{gathered}
$$

The solution of this element-by-element computation $\boldsymbol{u}_{h}^{*}$ converges with order $k+2$ in the $\mathcal{L}_{2}$ norm, see $[8,6]$ for a proof for standard HDG.

\subsection{On the stability of the X-HDG discretization}

The present formulation is subject to the usual deterioration of condition numbers in the linear system for very small cut scenarios, unless proper stabilization terms are used. The stability behavior is indeed similar to the Laplace case discussed in [14], as opposed to formulations using discretizations of the primal form where inf-sup stability needs to be specifically taken into account when developing stabilized formulations, see e.g. [3]. Due to the special construction of the cut scenario with an additional trace variable on the interface, the standard HDG a priori error estimation presented in [6] carries over to the cut case with our method. In particular, the representation of the velocity gradient $L$ as polynomials of degree $k$, the same as the pressure variable $p$, implies inf-sup stability also in elements cut by the interface. 


\section{X-HDG formulation for bimaterial problems}

Let $\Omega \subset \mathbb{R}^{d}$ be a bounded domain divided in two disjoint subdomains, $\bar{\Omega}=\bar{\Omega}_{1} \cup \bar{\Omega}_{2}$, with an interface $\mathcal{I}=\bar{\Omega}_{1} \cap \bar{\Omega}_{2}$. The following Stokes bimaterial problem is considered,

$$
\begin{aligned}
& -\boldsymbol{\nabla} \cdot(\nu \boldsymbol{\nabla u})+\nabla p=\boldsymbol{f} \quad \text { in } \Omega_{1} \cup \Omega_{2}, \\
& \boldsymbol{\nabla} \cdot \boldsymbol{u}=\mathbf{0} \quad \text { in } \Omega_{1} \cup \Omega_{2}, \\
& \llbracket \boldsymbol{u} \otimes \boldsymbol{n} \rrbracket=\mathbf{0} \quad \text { on } \mathcal{I}, \\
& \llbracket(-\nu \boldsymbol{\nabla} \boldsymbol{u}+p \mathbf{I}) \cdot \boldsymbol{n} \rrbracket=\boldsymbol{g} \quad \text { on } \mathcal{I}, \\
& \boldsymbol{u}=\boldsymbol{u}_{D} \quad \text { on } \partial \Omega,
\end{aligned}
$$

where the material viscosity may be discontinuous across the interface (that is, $\nu=\nu_{i}$ in $\Omega_{i}$ for $i=1,2$ ), and $\boldsymbol{g}$ is a given interface force. Again, Dirichlet boundary conditions are considered on the exterior boundary, $\partial \Omega$, for simplicity. An additional constraint on the pressure is added to ensure a unique solution, for instance, setting the mean of the pressure on the boundary of the first element to 0 or to a given constant.

The domain $\Omega$ is again assumed to be split into finite elements $\left\{K_{i}\right\}_{i=1}^{\mathrm{n}_{\mathrm{el}}}$, and $\Gamma$ is now the union of all faces in the mesh. Thus, the bimaterial Stokes problem (18) can be written as a set of local problems, and global equations. The local problem for elements not intersecting the interface is the standard HDG one (2a), whereas the local problem for an element cut by the interface now incorporates the interface conditions,

$$
\left.\begin{array}{rl}
\boldsymbol{L}-\boldsymbol{\nabla} \boldsymbol{u}=\mathbf{0}, \boldsymbol{\nabla} \cdot(-\nu \boldsymbol{L}+p \mathbf{I})=\boldsymbol{f}, \boldsymbol{\nabla} \cdot \boldsymbol{u} & =\mathbf{0} \text { in } K_{i} \\
\llbracket \boldsymbol{u} \otimes \boldsymbol{n} \rrbracket=\mathbf{0}, \llbracket(-\nu \nabla \boldsymbol{u}+p \mathbf{I}) \cdot \boldsymbol{n} \rrbracket & =\boldsymbol{g} \text { on } \mathcal{I}_{i} \\
\boldsymbol{u} & =\hat{\boldsymbol{u}} \text { on } \partial K_{i} \\
\frac{1}{\left|\partial K_{i}\right|} \int_{\partial K_{i}} p d S & =\rho_{i}
\end{array}\right\} \text { if } \mathcal{I}_{i}:=\mathcal{I} \cap K_{i} \neq \emptyset .
$$

The global problem is given by (3) with the solvability condition $\int_{\partial K_{i}} \hat{\boldsymbol{u}} \cdot \boldsymbol{n} d S=0$ for all elements, and the global constraint for the pressure $\rho_{1}=0$.

It is worth noting that the conservativity condition (3a) applies only to faces that do not match the bimaterial interface. If a face coincides with the interface, the conservativity condition with interface force, $\llbracket(-\nu \nabla \boldsymbol{u}+p \mathbf{I}) \cdot \boldsymbol{n} \rrbracket=\boldsymbol{g}$, should be considered.

The following discrete spaces for elemental variables, $\boldsymbol{u}, \boldsymbol{L}$ and $p$, and for the trace variable, $\hat{\boldsymbol{u}}$, are considered

$$
\begin{aligned}
& \mathcal{V}_{\mathrm{enr}}^{h}:=\left\{v \in \mathcal{L}^{2}(\Omega):\left.v\right|_{K_{i}} \in \mathcal{P}_{k}\left(K_{i}\right) \quad \text { if } K_{i} \cap \mathcal{I}=\emptyset,\right. \\
& \left.\left.v\right|_{K_{i}} \in \mathcal{P}_{k}\left(K_{i}\right) \oplus H \mathcal{P}_{k}\left(K_{i}\right) \text { if } K_{i} \cap \mathcal{I} \neq \emptyset\right\}, \\
& \Lambda_{\mathrm{enr}}^{h}:=\left\{\hat{v} \in \mathcal{L}^{2}(\Gamma):\left.\hat{v}\right|_{\Gamma_{i}} \in \mathcal{P}_{k}\left(\Gamma_{i}\right) \quad \text { if } \Gamma_{i} \cap \mathcal{I}=\emptyset\right. \text {, } \\
& \left.\left.\hat{v}\right|_{\Gamma_{i}} \in \mathcal{P}_{k}\left(\Gamma_{i}\right) \oplus H_{\mathcal{P}_{k}}\left(\Gamma_{i}\right) \text { if } \Gamma_{i} \cap \mathcal{I} \neq \emptyset\right\} .
\end{aligned}
$$

The Heaviside function $H$ enriches the approximation in cut elements and on cut faces and is defined as

$$
H=\left\{\begin{aligned}
1 & \text { in } \Omega_{1} \\
-1 & \text { in } \Omega_{2}
\end{aligned}\right.
$$

The X-HDG approximation space is discontinuous across the interface for the velocity $\boldsymbol{u}$, its gradient $\boldsymbol{L}$, and the pressure $p$. This is the natural choice for the enrichment of the gradient $\boldsymbol{L}$ and the pressure $p$ that are discontinuous across the interface. Despite the continuity of the velocity $\boldsymbol{u}$ in the problem setting (18), X-HDG considers a discontinuous approximation and imposes the condition on the solution $\boldsymbol{u}$ and on the tractions in the usual HDG fashion. 
For the sake of simplicity, all derivations in this section are done assuming that cut elements are split into two regions, which corresponds to the usual situation. Thus, one Heaviside enrichment is considered in (20). However, with high-order approximations more complicated situations, dividing elements or faces into more regions, may appear. If it is the case, an enrichment with multiple Heaviside functions should be considered to ensure an independent approximation in each region of the element or face; for details see [14].

\subsection{X-HDG for bimaterial Stokes: local problems}

The local problem for standard elements is again the same as for standard HDG (4). The XHDG local problem for an element intersected by the material interface is the discretization of (19), that is: given $\hat{\boldsymbol{u}}_{h} \in\left[\Lambda_{\mathrm{enr}}^{h}\right]^{d}$ and $\rho_{i} \in \mathbb{R}$, find $\boldsymbol{u}_{h} \in\left[\mathcal{P}_{k}\left(K_{i}\right) \oplus H \mathcal{P}_{k}\left(K_{i}\right)\right]^{d}, \boldsymbol{L}_{h} \in$ $\left[\mathcal{P}_{k}\left(K_{i}\right) \oplus H \mathcal{P}_{k}\left(K_{i}\right)\right]^{d \times d}, p_{h} \in \mathcal{P}_{k}\left(K_{i}\right) \oplus H \mathcal{P}_{k}\left(K_{i}\right)$ and $\widetilde{\boldsymbol{u}}_{h}^{i} \in\left[\mathcal{P}_{k}\left(\mathcal{I}_{i}\right)\right]^{d}$ such that

$$
\begin{aligned}
& \int_{K_{i}}\left(-\boldsymbol{\nabla} \cdot\left(\nu \boldsymbol{L}_{h}\right)+\nabla p_{h}\right) \cdot \boldsymbol{v} d V+\int_{\partial K_{i}} \nu \tau\left(\boldsymbol{u}_{h}-\hat{\boldsymbol{u}}_{h}\right) \cdot \boldsymbol{v} d S \\
& +2 \int_{\mathcal{I}_{i}}\left(\left\{\tau \nu \boldsymbol{u}_{h}\right\}-\{\tau \nu\} \widetilde{\boldsymbol{u}}_{h}^{i}\right) \cdot \boldsymbol{v} d S=\int_{K_{i}} \boldsymbol{f} \cdot \boldsymbol{v} d V \\
& \int_{K_{i}} \boldsymbol{L}_{h}: \boldsymbol{Q} d V+\int_{K_{i}}(\boldsymbol{\nabla} \cdot \boldsymbol{Q}) \cdot \boldsymbol{u}_{h} d V-\int_{\partial K_{i}}(\boldsymbol{Q} \cdot \boldsymbol{n}) \cdot \hat{\boldsymbol{u}}_{h} d S-\int_{\mathcal{I}_{i}} \llbracket \boldsymbol{Q} \cdot \boldsymbol{n} \rrbracket \cdot \widetilde{\boldsymbol{u}}_{h}^{i} d S=0, \\
& \int_{K_{i}} \boldsymbol{u}_{h} \cdot \nabla q d V-\int_{\partial K_{i}}\left(\hat{\boldsymbol{u}}_{h} \cdot \boldsymbol{n}\right) q d S-\int_{\mathcal{I}_{i}} \llbracket q \boldsymbol{n} \rrbracket \cdot \widetilde{\boldsymbol{u}}_{h}^{i} d S=0, \\
& \frac{1}{\left|\partial K_{i}\right|} \int_{\partial K_{i}} p_{h} d S=\rho_{i} \\
& \int_{\mathcal{I}_{i}} \llbracket\left(-\nu \boldsymbol{L}_{h} \cdot \boldsymbol{n}+p_{h} \boldsymbol{n}\right) \rrbracket \cdot \widetilde{\boldsymbol{v}} d S+2 \int_{\mathcal{I}_{i}}\left(\left\{\tau \nu \boldsymbol{u}_{h}\right\}-\{\tau \nu\} \widetilde{\boldsymbol{u}}_{h}^{i}\right) \cdot \widetilde{\boldsymbol{v}} d S=\int_{\mathcal{I}_{i}} \boldsymbol{g} \cdot \widetilde{\boldsymbol{v}} d S
\end{aligned}
$$

holds for all $\boldsymbol{v} \in\left[\mathcal{P}_{k}\left(K_{i}\right) \oplus H \mathcal{P}_{k}\left(K_{i}\right)\right]^{d}, \boldsymbol{Q} \in\left[\mathcal{P}_{k}\left(K_{i}\right) \oplus H \mathcal{P}_{k}\left(K_{i}\right)\right]^{d \times d}, q \in \mathcal{P}_{k}\left(K_{i}\right) \oplus$ $H \mathcal{P}_{k}\left(K_{i}\right)$ and $\widetilde{\boldsymbol{v}} \in\left[\mathcal{P}_{k}\left(\mathcal{I}_{i}\right)\right]^{d}$.

Again a new trace variable $\widetilde{\boldsymbol{u}}_{h}^{i}$ is introduced to approximate the trace of the velocity on the interface. With this auxiliary variable, the standard HDG local problem can be stated in $\Omega_{1} \cap K_{i}$ and in $\Omega_{2} \cap K_{i}$ separately, setting the Dirichlet condition to $\hat{\boldsymbol{u}}$ on $\partial \Omega_{1} \backslash I_{i}$ and on $\partial \Omega_{2} \backslash I_{i}$, and to the new trace variable $\widetilde{\boldsymbol{u}}_{h}^{i}$ on the interface $\mathcal{I}_{i}$. Summing up the two local problems results in equations (21a) and (21b). Finally, equation (21c) is the discretization of the conservativity condition across the interface, $\llbracket(-\nu \boldsymbol{\nabla} \boldsymbol{u}+p \mathbf{I}) \cdot \boldsymbol{n} \rrbracket=\boldsymbol{g}$ on $\mathcal{I}_{i}$, with the numerical velocity gradient (8).

A proper numerical quadrature must be considered for the numerical integration in the element, accounting for the discontinuous nature of the approximations and the material parameter $\nu$. Again, the $r$-th degree parametrization of the interface $\mathcal{I}_{i}$ discussed in Section 4 is considered for the definition of numerical quadratures in each portion of the element, for numerical integration on the interface $\mathcal{I}_{i}$, and for the definition of the approximation space for the trace on the interface $\mathcal{P}_{k}\left(\mathcal{I}_{i}\right)$. 
Considering nodal basis functions and imposing the constraint on the pressure (21b) with a Lagrange multiplier $\lambda$, the local problem (21) can be written in matrix form as

$$
\left[\begin{array}{ccccc}
\mathbf{A}_{u u}^{i} & \mathbf{A}_{u L}^{i} & \mathbf{A}_{u p}^{i} & \mathbf{A}_{u \widetilde{u}}^{i} & \mathbf{0} \\
\mathbf{A}_{L u}^{i} & \mathbf{A}_{L L}^{i} & \mathbf{0} & \mathbf{A}_{L \widetilde{u}}^{i} & \mathbf{0} \\
\mathbf{A}_{p u}^{i} & \mathbf{0} & \mathbf{0} & \mathbf{A}_{p \widetilde{u}}^{i} & \mathbf{A}_{\rho p}^{i} T \\
\mathbf{A}_{\widetilde{u} u}^{i} & \mathbf{A}_{\widetilde{u} L}^{i} & \mathbf{A}_{\widetilde{u} p}^{i} & \mathbf{A}_{\widetilde{u} \widetilde{u}}^{i} & \mathbf{0} \\
\mathbf{0} & \mathbf{0} & \mathbf{A}_{\rho p}^{i} & \mathbf{0} & \mathbf{0}
\end{array}\right]\left[\begin{array}{c}
\mathbf{u}^{i} \\
\mathbf{L}^{i} \\
\mathbf{p}^{i} \\
\widetilde{\mathbf{u}}^{i} \\
\lambda
\end{array}\right]=\left[\begin{array}{c}
\mathbf{f}_{u}^{i} \\
\mathbf{0} \\
\mathbf{0} \\
\mathbf{f}_{\widetilde{u}}^{i} \\
\mathbf{0}
\end{array}\right]+\left[\begin{array}{c}
\mathbf{0} \\
\mathbf{0} \\
\mathbf{0} \\
\mathbf{0} \\
\mathbf{1}
\end{array}\right] \rho_{i}-\left[\begin{array}{c}
\mathbf{A}_{u \widehat{u}}^{i} \\
\mathbf{A}_{L \widehat{u}}^{i} \\
\mathbf{A}_{p \widehat{u}}^{i} \\
\mathbf{0} \\
\mathbf{0}
\end{array}\right] \mathbf{\Lambda}^{i}
$$

This system leads to a local solver of the form (10) expressing $\mathbf{u}^{i}, \mathbf{L}^{i}$ and $\mathbf{p}^{i}$ in terms of the nodal values of the trace of the velocity, $\Lambda^{i}$, and the mean of the pressure in the boundary of the elements, $\rho_{i}$. Similarly to the formulation with Neumann interfaces, the auxiliary trace variable $\widetilde{\boldsymbol{u}}^{i}$ is a variable in the local problems, that does not appear in the global problem.

\subsection{X-HDG for bimaterial Stokes: global problem}

The X-HDG global problem, corresponding to the discretization of (3) with the solvability conditions and the global constraint for the pressure is to find $\hat{\boldsymbol{u}}_{h} \in\left[\Lambda_{\mathrm{enr}}^{h}\right]^{d}$ and $\left\{\rho^{i}\right\}_{i=1}^{\mathrm{n}_{\mathrm{el}}}$ satisfying (12) for all $\widehat{\boldsymbol{v}} \in\left\{\left[\Lambda_{\mathrm{enr}}^{h}\right]^{d}: \widehat{\boldsymbol{v}}=0\right.$ on $\left.\partial \Omega\right\}, \int_{\partial K_{i}} \hat{\boldsymbol{u}}_{h} \cdot \boldsymbol{n} d S=0$ for $i=1, \ldots, \mathrm{n}_{\mathrm{el}}$, $\hat{\boldsymbol{u}}_{h}=\mathbb{P}_{2}\left(\boldsymbol{u}_{D}\right)$ on $\partial \Omega$ and $\rho_{1}=0$, where $\boldsymbol{u}_{h}, \boldsymbol{L}_{h}$ and $p_{h}$ solve the local problem for each element, i.e., (4) or (21).

These equations are exactly the same as for standard HDG, but with enriched approximation spaces for cut elements and cut faces. They can be written in matrix form as (15) for $f=1, \ldots, \mathrm{n}_{\mathrm{fc}}$, and $\mathbf{A}_{\rho \widehat{u}} \boldsymbol{\Lambda}^{i}=0$ for $i=1, \ldots, \mathrm{n}_{\mathrm{el}}$.

Similarly to standard HDG, inserting the result of the local solvers in the global equations leads to a linear system of equations involving only the mean of the pressure on the boundary of the elements $\left\{\rho_{i}\right\}_{i=1}^{n_{\text {el }}}$ and the trace of the velocity $\left\{\widehat{\boldsymbol{u}}^{f}\right\}_{f=1}^{\mathrm{n}_{\mathrm{e} 1}}$, but with double degrees of freedom for enriched faces.

\subsection{X-HDG for bimaterial Stokes: postprocess for superconvergent solution}

The element-by-element postprocessing to obtain a superconvergent solution in every cut element $K_{i}$ with $K_{i} \cap \mathcal{I} \neq \emptyset$ is: given $\boldsymbol{u}_{h} \in\left[\mathcal{P}_{k}\left(K_{i}\right) \oplus H \mathcal{P}_{k}\left(K_{i}\right)\right]^{d}$ and $\boldsymbol{L}_{h} \in\left[\mathcal{P}_{k}\left(K_{i}\right) \oplus\right.$ $\left.H \mathcal{P}_{k}\left(K_{i}\right)\right]^{d \times d}$, find $\boldsymbol{u}_{h}^{*} \in\left[\mathcal{P}_{k+1}\left(K_{i}\right) \oplus H \mathcal{P}_{k+1}\left(K_{i}\right)\right]^{d}$ such that

$$
\begin{gathered}
\int_{\Omega_{i}} \boldsymbol{\nabla} \boldsymbol{u}_{h}^{*}: \nabla \boldsymbol{v} d V=\int_{K_{i}} \boldsymbol{L}_{h}: \nabla \boldsymbol{v} d V \quad \forall \boldsymbol{v} \in\left[\mathcal{P}_{k+1}\left(K_{i}\right) \oplus H \mathcal{P}_{k+1}\left(K_{i}\right)\right]^{d} \\
\int_{\Omega_{i}} \boldsymbol{u}_{h}^{*} d V=\int_{\Omega_{i}} \boldsymbol{u}_{h} d V \\
\int_{\Omega_{i}} H \boldsymbol{u}_{h}^{*} d V=\int_{\Omega_{i}} H \boldsymbol{u}_{h} d V
\end{gathered}
$$

This elemental postprocess is equivalent to consider the usual postprocessing procedure (17) on each one of the material domains, $K_{i} \cap \Omega_{1}$ and $K_{i} \cap \Omega_{2}$. 


\section{Level-set representation of interfaces and approximation}

Following the X-FEM philosophy, interfaces are assumed to be defined with level-set functions given by nodal values on an $r$-th degree mesh, using the same elements as the computational mesh and degree $r \geq k$.

Integrals in cut elements $\Omega_{i}$ and on interfaces require quadrature that is restricted to the portion of the elements intersecting with the actual computational domain. To keep highorder convergence rates, a specific representation of the interface $\mathcal{I}_{i}:=\mathcal{I} \cap K_{i}$ in cut elements is necessary. For 2D computations, the $r$-degree representation proposed in $[4,15]$ is considered here: to define an $r$-th degree parametrization, $r+1$ points are computed on the interface $\mathcal{I}_{i}$. In fact, the usual isoparametric transformation (superparametric transformation for $r>k$ ) from the 1D reference element $(-1,1)$ to the physical curved segment defined by the $r+1$ nodes, is applied, see an example in Figure 2 (right). This representation of the interface leads to proper definitions of numerical quadratures in the domain $\Omega_{i}$ and on the interface $\mathcal{I}_{i}$.

In addition, this parametrization is also used to define the approximation space for the trace on the interface: $\mathcal{P}_{k}\left(\mathcal{I}_{i}\right)$ is generated by the transformation of the nodal polynomial basis in $(-1,1)$ to the curved segment, approximating $\mathcal{I}_{i}$ by means of the usual isoparametric transformation given by the coordinates of the $r+1$ nodes on $\mathcal{I}_{i}$.

A similar strategy could be considered for 3D computations. Points on the interface are computed to define an $r$-th degree parametrization of the interface of the element, which is a curved triangle or a quadrilateral. The parametrization induces a parametrization of the two regions of the volume, which may be split in sub-cells for simplicity. These parametrizations lead to numerical quadratures for the interface and for the volumes, see [10] for details, in the context of X-FEM. In addition, the parametrization is used as transformation from the triangle or quadrilateral reference element to the physical curved interface for X-HDG, defining the approximation space for the trace variable as the transformation of the 2D standard polynomial basis.

Only 2D numerical tests are considered in this manuscript. In all cases, a $k$-th degree level set function is used for the X-HDG solution and an additional level set function with polynomial degree $r=k+1$ is used for the postprocessed solution. Nevertheless, it is important mentioning that higher degrees for the level-set function may be necessary to ensure high-order convergence rates for bimaterial interfaces with involved interface geometries. For instance, the heat problem with a kidney-shaped interface in [14] requires degree $r=k+2$ to show optimal convergence. According to the analysis for standard HDG with meshes fitted to the interface in [18], a level set function with degree $r=2 k+1$ provides optimal convergence of X-HDG in the more general situation for bimaterial problems.

\section{Numerical examples}

The performance of X-HDG is assessed on several 2D numerical examples involving Neumann, Dirichlet, or material interface conditions. In all tests, the stabilization parameter is set to $\tau=1$ over all faces. A $k$-th degree level set function is used for the X-HDG solution and an additional level set function with polynomial degree $r=k+1$ is used for the postprocessed solution. 

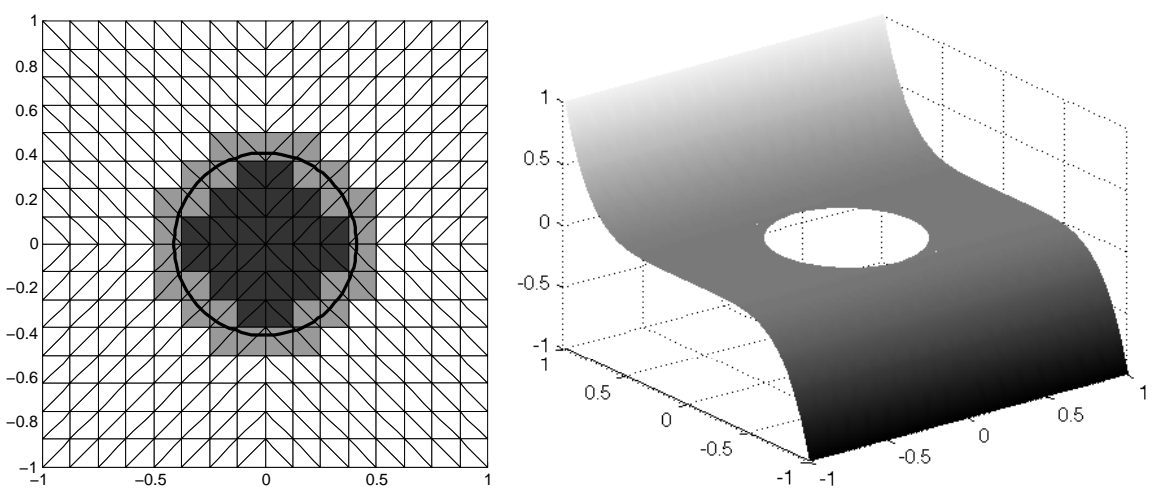

Fig. 3 Circular void: computational mesh after two mesh refinements and circular interface with radius $\mathrm{R}=0.41$ (left), and $x$ component of the velocity (right). White elements are in the interior of the domain. Elements in light gray are elements cut by the interface $\mathcal{I}$. Elements in dark gray are inside the void and disregarded in the computation.
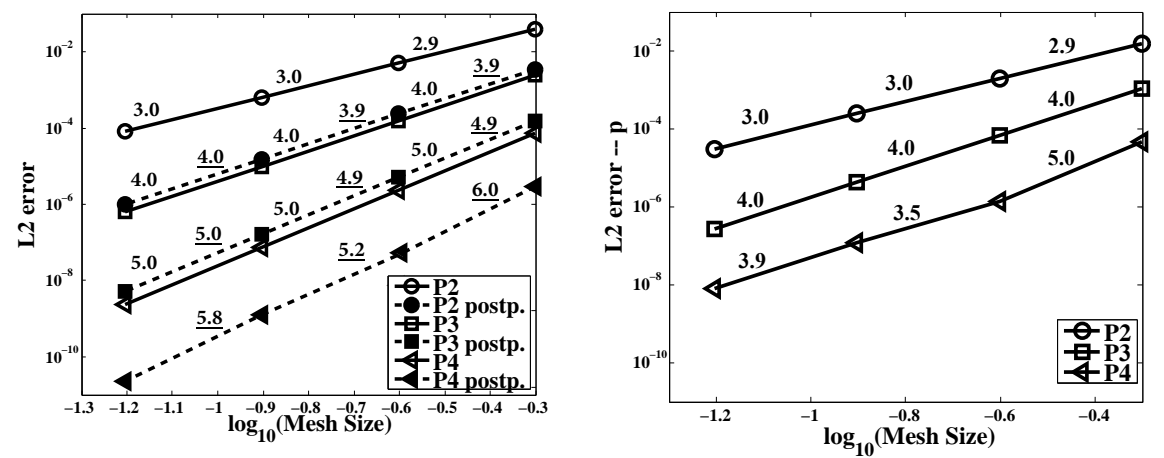

Fig. 4 Circular void: convergence history of X-HDG velocity (left) and pressure (right), with Dirichlet boundary conditions on the interface and radius $R=0.41$. The numbers correspond to the slope of each segment, and are underlined for postprocessed velocity.

\subsection{Circular void}

The Stokes problem (1) with $\nu=1$ is solved over a square domain with a centered circular void of radius $R$, that is, $\Omega=(-1,1)^{2} \backslash B((0,0), R)$. We consider both Neumann and Dirichlet boundary conditions on the void boundary $\mathcal{I}=\partial B((0,0), R)$, while Dirichlet boundary conditions are considered on the exterior boundary $\partial \Omega \backslash \mathcal{I}=\partial\left((0,1)^{2}\right)$. The analytical solution is a smooth polynomial solution,

$$
\boldsymbol{u}(x, y)=\left(y^{5}, x^{5}\right)^{T}, \quad p=x+y .
$$

The right hand side $f$ is chosen to match with this analyical solution. The computational domain after two uniform refinements of the mesh as well as the $x$ component of the velocity are shown in Figure 3. As stated in section 2, the computational mesh covers the domain $\Omega$ and fits to the exterior boundary $\partial \Omega \backslash \mathcal{I}$. A level-set function is used to describe the interface $\mathcal{I}$. In all mesh representations, elements filled with white color are in the interior of the domain, elements in light gray are elements cut by the interface $\mathcal{I}$, and elements in dark gray are inside the void and hence disregarded in the computation. 

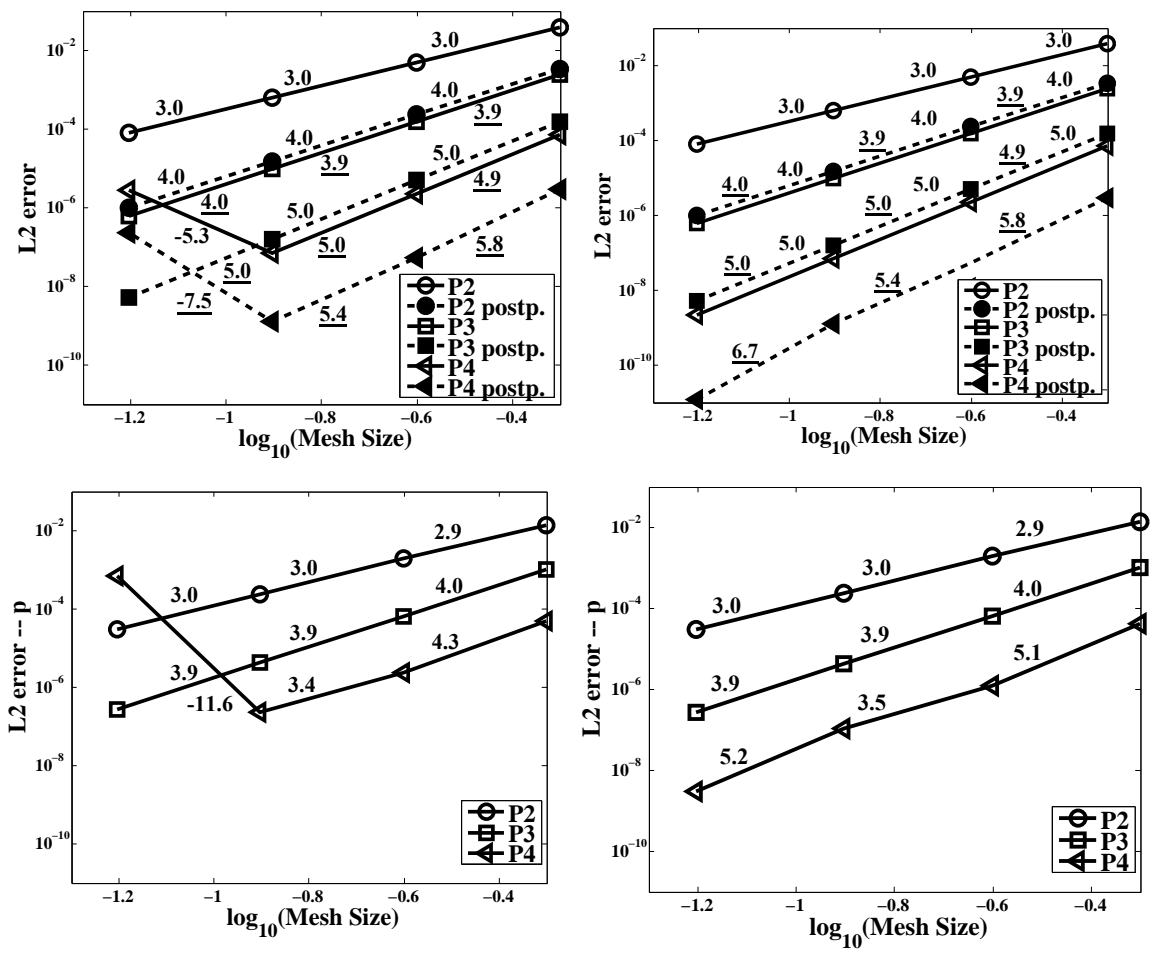

Fig. 5 Circular void: convergence history of X-HDG velocity (top) and pressure (bottom), with Neumann boundary conditions on the interface and radius $R=0.5$, with uniform regular mesh (left) and with a slightly modified the mesh to avoid ill-conditioning (right). The numbers correspond to the slope of each segment, and are underlined for postprocessed velocity.

Figure 4 shows the evolution of the $\mathcal{L}_{2}$ error for the velocity and the postprocessed superconvergent velocity (left) and for the pressure (right), for polynomial degrees $k=2,3,4$ and 3 nested mesh refinement steps. Dirichlet boundary conditions are imposed on the circle. This toy example verifies the optimal convergence rates of X-HDG without mesh fitting. In this test, the radius of the circle has been selected to avoid ill-conditioning problems, leading to optimal convergence rates for the velocity approximation, including superconvergence for the post-processed velocity. However, ill-conditioning effects can be observed in the pressure convergence plot for the finer meshes and the highest degree, $k=4$.

Ill-conditionting problems can be avoided, for instance, by using the strategy described in [14] that slightly modifies the mesh in the presence of bad-cut elements, that is, elements cut by the interface with a small support inside the computational domain. Figures 5 and 6 illustrate the strategy. Figure 5 shows the convergence plots for velocity and pressure, on a uniform regular mesh (left column) and a slightly modified mesh (right column), for a circular interface with radius $R=0.5$. In this case Neumann boundary conditions are imposed on the circle. The presence of sliver cut elements in the uniform regular mesh case with $R=0.5$ leads to severe ill-conditioning of the matrix, spoiling the convergence for the finer meshes and higher degrees. Figure 6 shows two details of the computational mesh, depicting the elements that are cut by the interface in light gray. In the top left zoom, three elements become very slow once they are cut, because they have one vertex very close to 

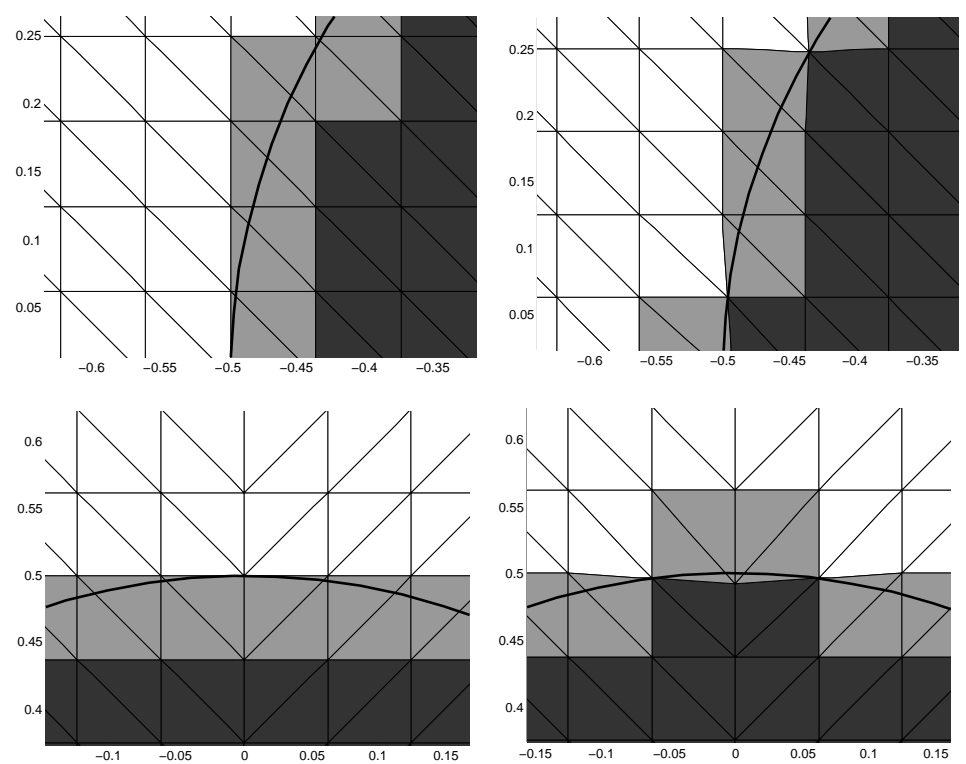

Fig. 6 Circular void: slight modification of the mesh to avoid ill-conditioning (right) for the interface with $R=0.5$. Details of the mesh before (left) and after (right) the modification. Light gray elements are cut by the interface.

the interface. These nodes are moved on top of the interface such that the corresponding elements are not cut elements anymore. The bottom left zoom shows two elements with a face close to the interface, again in elements with a very small overlap with the domain. In [14] those faces were moved to fit the interface, and the corresponding elements became noncut elements with a curved edge fitting to the interface. Here, to avoid curved edges, the node closer to the interface is moved farther away from it, ensuring that the corresponding cut elements have a large enough overlap with the computational domain. Both strategies lead to better conditioning and recover optimal convergence rates for both velocity and pressure.

\subsection{Kidney shaped void with Neumann conditions}

The square domain $(-1,1)^{2}$ is cropped by the kidney-shaped interface defined as the 0-level set of

$$
\varphi(x, y)=\left(3\left((x+0.5)^{2}+y^{2}\right)-x-0.5\right)^{2}-\left((x+0.5)^{2}+y^{2}\right)+0.1,
$$

as shown in the left panel of Figure 7. Neumann boundary conditions are considered at the interface, and Dirichlet conditions are imposed on the exterior boundary. The analytical solution reads

$$
\boldsymbol{u}(x, y)=\left(0, \cos \left(1-x^{2}\right)\right)^{T}, \quad p=x+y .
$$

Computations are done for polynomial degrees $k=1, \ldots, 4$, and 3 nested mesh refinement steps. Even though this test considers an involved geometry, which may require higher degrees for the level-set function (see [14]), a level-set representation with the same degree as 

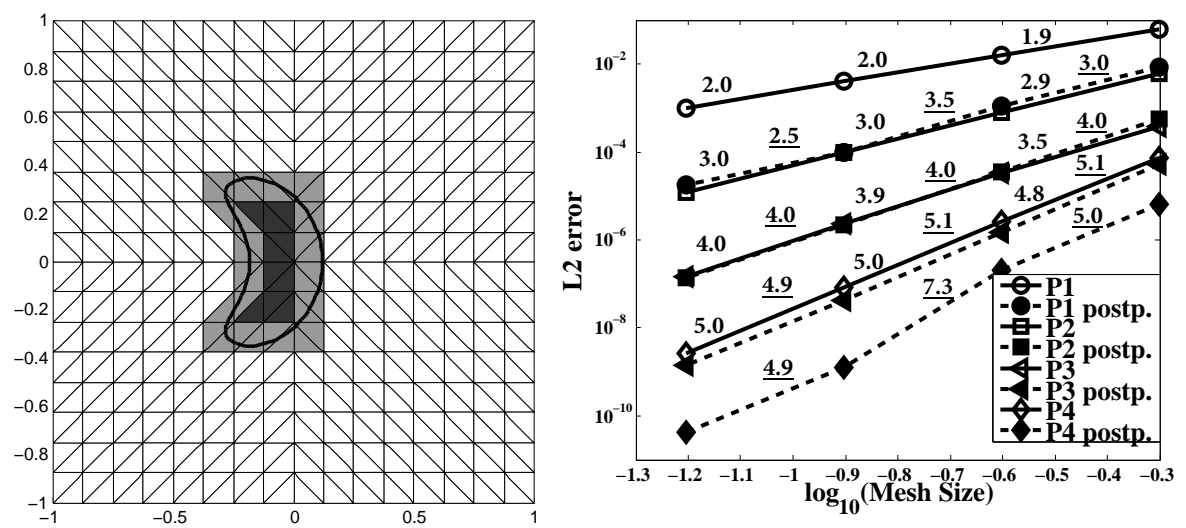

Fig. 7 Kidney shaped void with Neumann conditions: computational mesh after two mesh refinements and kidney-shaped interface (left) and convergence of X-HDG velocity (right). Slopes corresponding to the post processed solution $\boldsymbol{u}^{*}$ are underlined.

the approximation is used here for the computation of the solution and of the superconvergent velocity.

Figure 7 right plots the evolution of the velocity $\mathcal{L}_{2}$ error, showing that X-HDG provides optimal convergence rates and superconvergence also for a rather complex interface all the way to errors below $10^{-10}$.

\subsection{Bimaterial straight interface}

Inspired by [17], a bimaterial problem with continuous solution is solved over a square domain $\Omega=(-0.4,1.6)^{2}$. The interface is a straight line passing through $\{y=0\}$, and material parameters are set to $\nu_{1}=1$ in $\Omega_{1}=\{(x, y) \in \Omega \mid y<0\}$, and to $\nu_{2}=2$ in $\Omega_{2}=\Omega \backslash \bar{\Omega}_{1}$, see the left panel of Figure 8 . Dirichlet boundary conditions are applied on the boundary $\partial \Omega$ and the analytical solution is

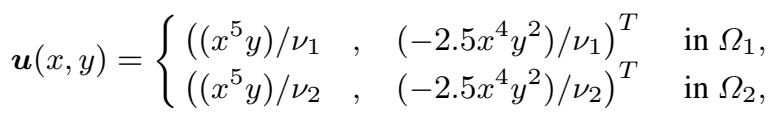

with $p=x+y$ in $\Omega$, see Figure 9 .

The right panel of Figure 8 shows the $\mathcal{L}_{2}$ error in the velocity for polynomial degrees $k=1, \ldots, 4$ and 3 nested steps of refinement. The results show that the X-HDG formulation for the bimaterial Stokes problems provides optimal convergence in the solution and superconvergence in the post-processed solution for problems including a straight interface.

\subsection{Bimaterial circular interface}

After testing the performance of X-HDG with voids and straight material interfaces, $\mathrm{X}-\mathrm{HDG}$ is examined next over a bimaterial problem where the interface is defined by a seconddegree level-set function. The Stokes problem (18) is solved over the square domain $\Omega=$ $(-1,1)^{2}$. A circular interface of radius $R=0.41$ divides the domain into two regions, 

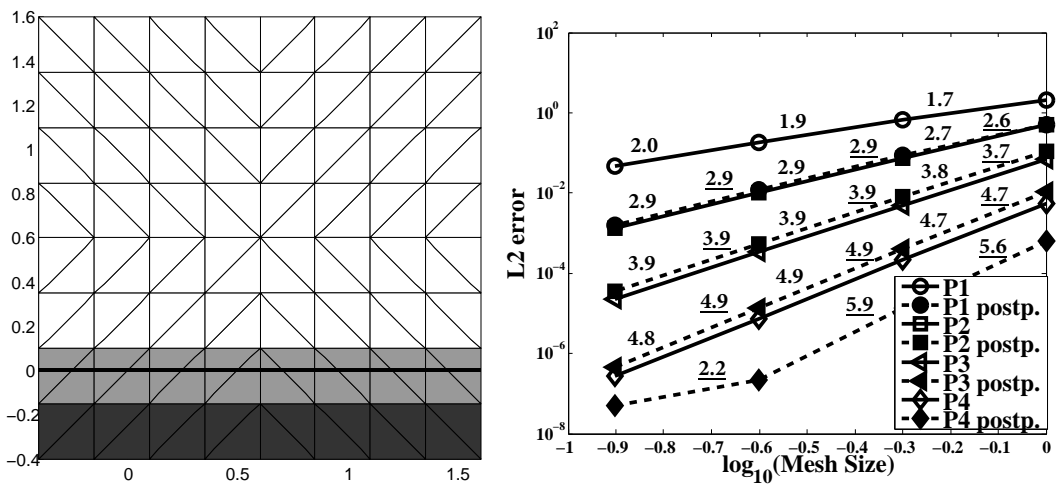

Fig. 8 Bimaterial straight interface: computational mesh after two mesh refinements and linear interface (left), and convergence for velocity (right).
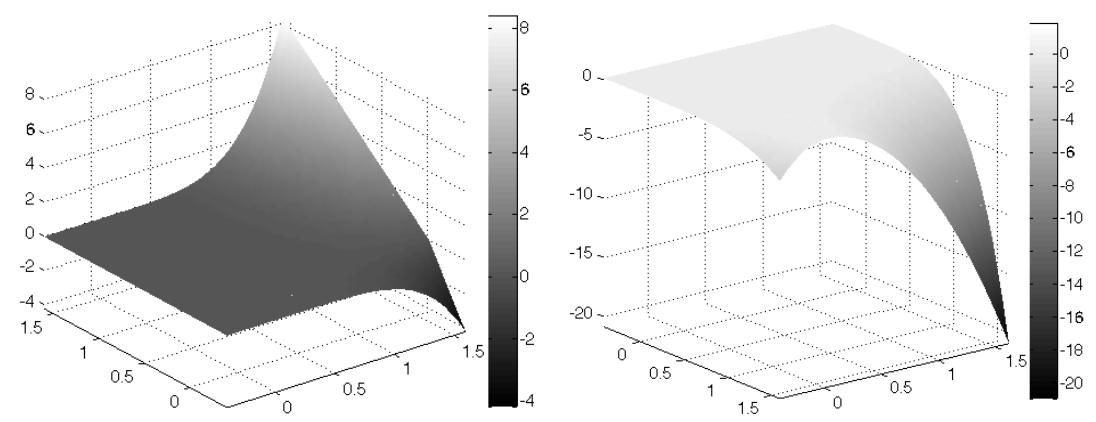

Fig. 9 Bimaterial straight interface: $x$ and $y$ components of the velocity.

$\Omega_{1}=\{\boldsymbol{x} \in \Omega \mid\|\boldsymbol{x}\|>R\}$ and $\Omega_{2}=\Omega \backslash \bar{\Omega}_{1}$, with material parameters $\nu_{1}=10$ and $\nu_{2}=1$, respectively, see Figure 10. The source term and the Dirichlet data on the exterior boundary are set to yield the analytical solution

$$
\boldsymbol{u}(x, y)= \begin{cases}\left(y^{5}, x^{5}\right)^{T} & \text { in } \Omega_{1}, \\ \left(\cos \left(1-y^{5}\right), \sin \left(1-x^{5}\right)\right)^{T} & \text { in } \Omega_{2},\end{cases}
$$

and $p=x+y$ in $\Omega$. In this setting, both the velocity and the tractions are discontinuous across the interface.

For this test, the interface conditions for the local problem in a cut element (19) are given by

$$
\begin{aligned}
\boldsymbol{u}_{1}-\boldsymbol{u}_{2}=\boldsymbol{\alpha} & \text { on } \mathcal{I}_{i}, \\
\llbracket(-\nu \nabla \boldsymbol{u}+p \mathbf{I}) \cdot \boldsymbol{n} \rrbracket=\boldsymbol{g} & \text { on } \mathcal{I}_{i}
\end{aligned}
$$

where $\boldsymbol{u}_{i}$ denotes the velocity from $\Omega_{i}$, and $\boldsymbol{\alpha}$ and $\boldsymbol{g}$ are data consistent with the analytical solution. The H-XDG discretization of the local problem (21a) is then modified with a new definition of the auxiliary trace variable, so that $\boldsymbol{u}_{1}=\widetilde{\boldsymbol{u}}_{h}^{i}+\boldsymbol{\alpha}$ and $\boldsymbol{u}_{2}=\widetilde{\boldsymbol{u}}_{h}^{i}$. The integrals $\int_{\mathcal{I}_{i}} \tau_{1} \nu_{1} \boldsymbol{\alpha} \cdot \boldsymbol{v} d S, \int_{\mathcal{I}_{i}}\left(\boldsymbol{Q}_{1} \cdot \boldsymbol{n}_{1}\right) \cdot \boldsymbol{\alpha} d S$ and $\int_{\mathcal{I}_{i}} q_{1} \boldsymbol{n}_{1} \cdot \boldsymbol{\alpha} d S$ are added to the right-hand side of the equations (21a), and the integral $\int_{\mathcal{I}_{i}} \tau_{1} \nu_{1} \boldsymbol{\alpha} \cdot \widetilde{\boldsymbol{v}} d S$ is added to the right-hand side of (21c). 

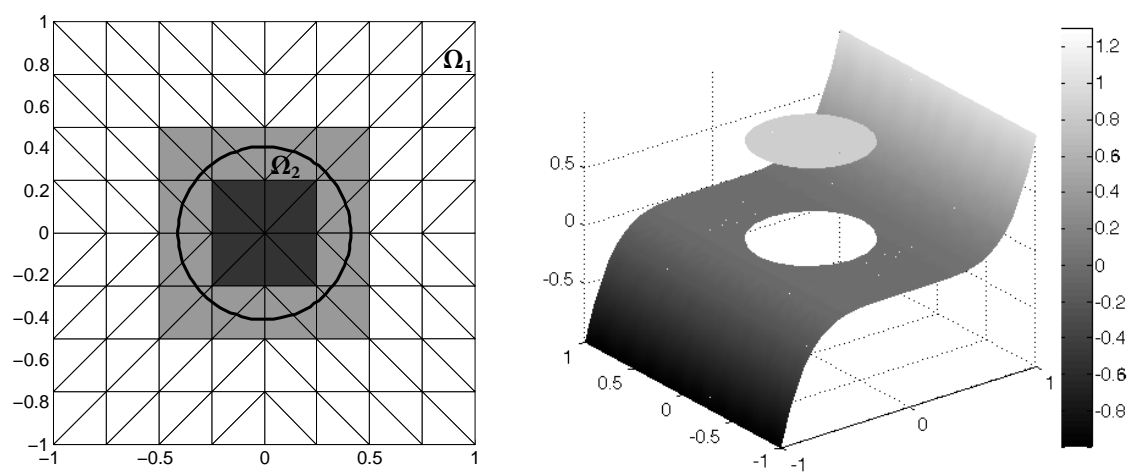

Fig. 10 Bimaterial circular interface: computational mesh after two mesh refinement steps and circular interface (left), and the $y$ component of the discontinuous velocity (right).
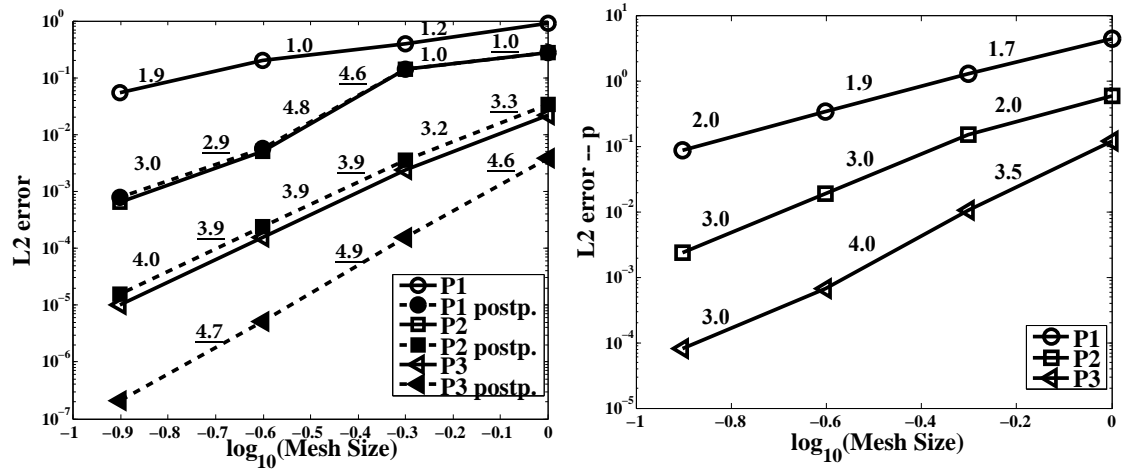

Fig. 11 Bimaterial circular interface: convergence plot for velocity (left) and pressure (right).

Figure 11 presents the convergence plots for the velocity, the postprocessed superconvergent velocity and pressure, demonstrating good agreement with the theoretical convergence rates.

\subsection{Stationary air bubble}

As a next example, the bimaterial Stokes problem defined in (18) is considered to model a realistic case: an air bubble with radius $R=2 / 3 \mathrm{~mm}$ in an ambient water domain $\Omega=$ $[-1,1]^{2}$, assuming a characteristic length $L=1 \mathrm{~mm}$ and a characteristic velocity $U=10^{-2}$ $\mathrm{m} / \mathrm{s}$. The non-dimensional viscosities are set to be $\nu_{1}=10^{-1}$ for water and $\nu_{2}=10^{-3}$ for the air bubble. The pressure jump at the interface is induced by an artificial surface tension force $f_{\mathcal{I}}(v)=\int_{\mathcal{I}} \tau \kappa v \cdot \boldsymbol{n} d S$ with surface tension coefficient $\tau_{s}=700$. In this formula, $\kappa(\boldsymbol{x})$ denotes the local curvature of the interface, thus, $\tau_{s} \kappa=\tau_{s} \frac{2}{R}=2100$. The analytical solution of the air bubble problem is given in [21], that is,

$$
\boldsymbol{u}(x, y)= \begin{cases}\left(-y \alpha(r) e^{-r^{2}}, x \alpha(r) e^{-r^{2}}\right)^{T} & \text { in } \Omega_{1}, \\ \left(-y \alpha(r) e^{-r^{2}}, x \alpha(r) e^{-r^{2}}\right)^{T} & \text { in } \Omega_{2}\end{cases}
$$



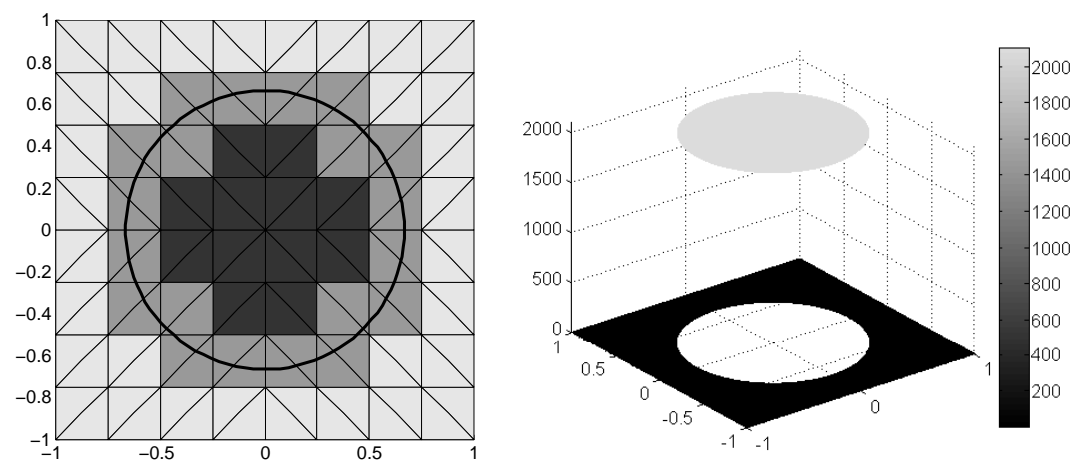

Fig. 12 Stationary air bubble: computational mesh after two mesh refinements and interface of the circular bubble (left), and pressure plot (right).
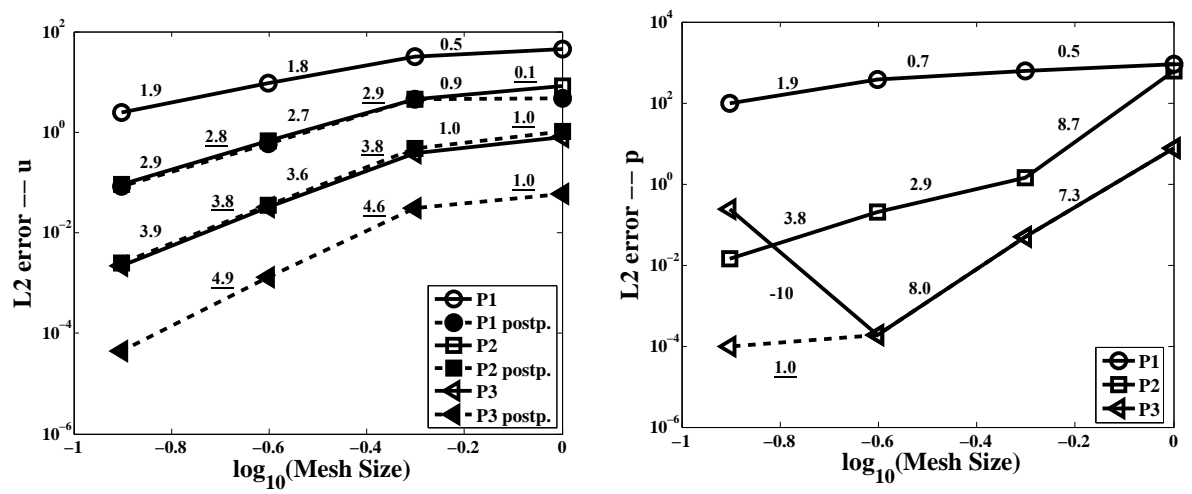

Fig. 13 Stationary air bubble: convergence plots over three refined meshes and varying approximation degrees for velocity (left). Convergence plots for pressure with (dashed line) and without (solid line) mesh modification (right).

with

$$
\alpha(r)= \begin{cases}\frac{1}{\nu_{1}}+\left(\frac{1}{\nu_{2}}-\frac{1}{\nu_{1}}\right) e^{r^{2}-R^{2}} & \text { in } \Omega_{1}, \\ \frac{1}{\nu_{2}} & \text { in } \Omega_{2},\end{cases}
$$

and

$$
p(x, y)= \begin{cases}x^{3} & \text { in } \Omega_{1}, \\ x^{3}+2 \tau_{s} / R & \text { in } \Omega_{2} .\end{cases}
$$

Figure 12 shows the air bubble and the water domain together with a plot of the discontinuous pressure. Figure 13 left panel presents the optimal convergence plots for the velocity and the postprocessed superconvergent velocity. Over the same figure on right panel, the pressure convergence is detailed. It can be observed that pressure convergence is more sensitive to the ill conditioning than the velocity, causing negative slopes. To avoid that, for the highest approximation degree and the finest mesh, we have employed the mesh modification strategy detailed earlier in Section 5.1. Although mesh modification alleviates the 

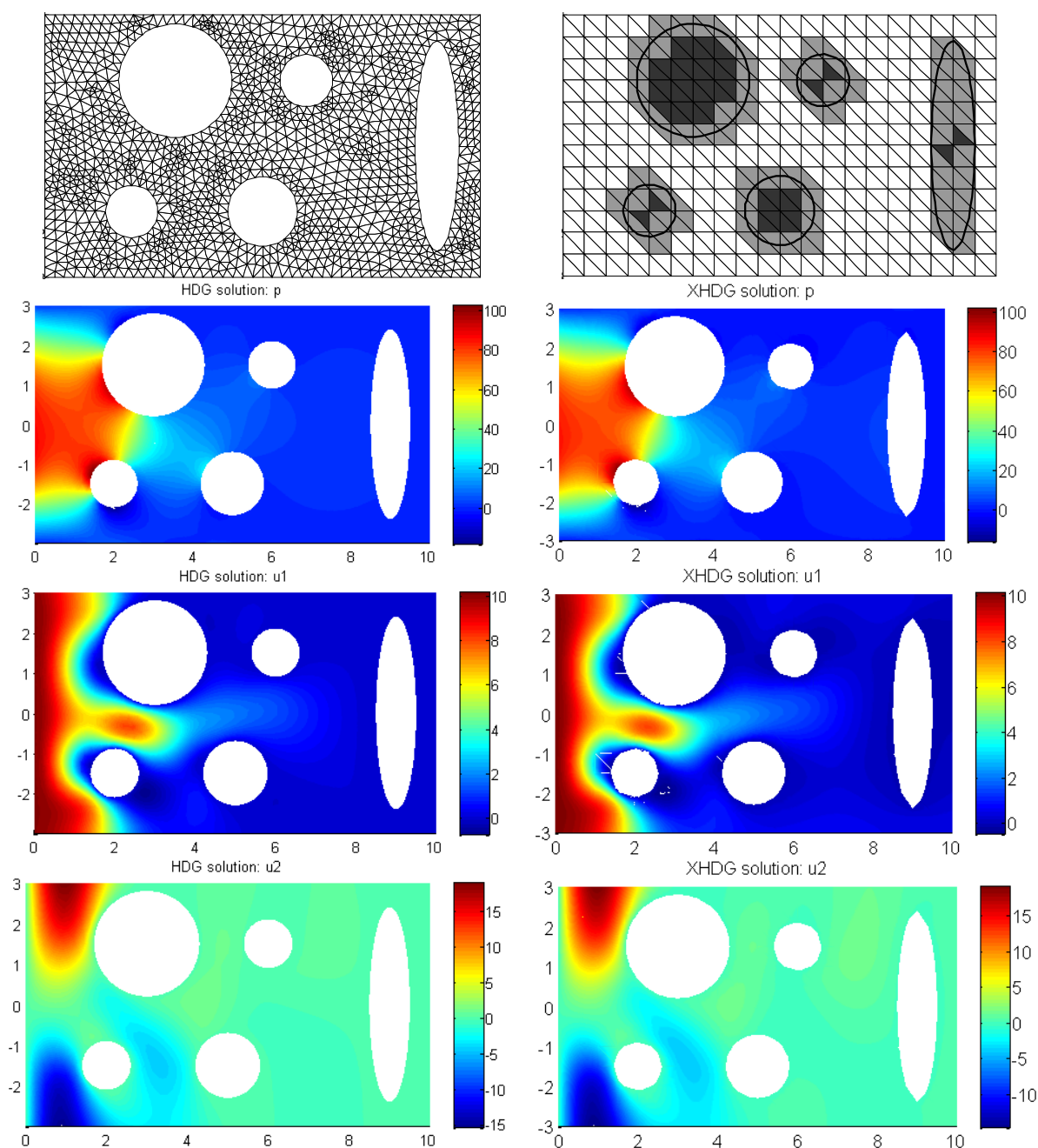

Fig. 14 Flow around several obstacles: computational mesh, pressure and velocity for HDG with a fine fitted mesh (left) and for X-HDG with uniform regular mesh (right)

ill conditioning and improves convergence, asymptotic rates are still not reached with the meshes considered in this analysis.

\subsection{Flow around several obstacles}

As a last example, the flow around several obstacles in a rectangular domain is studied. The obstacles are treated as voids with no-slip boundary conditions, $\boldsymbol{u}=\mathbf{0}$. An inflow condition is specified on the left of the computational domain with velocity $\boldsymbol{u}=(10,0)^{T}$ and homogeneous Neumann boundary conditions are imposed in the rest of the boundary. Figure 14 shows the computational mesh, the pressure, and the velocity components for HDG with a fine fitted mesh (left), considered as a reference solution, and X-HDG with a 

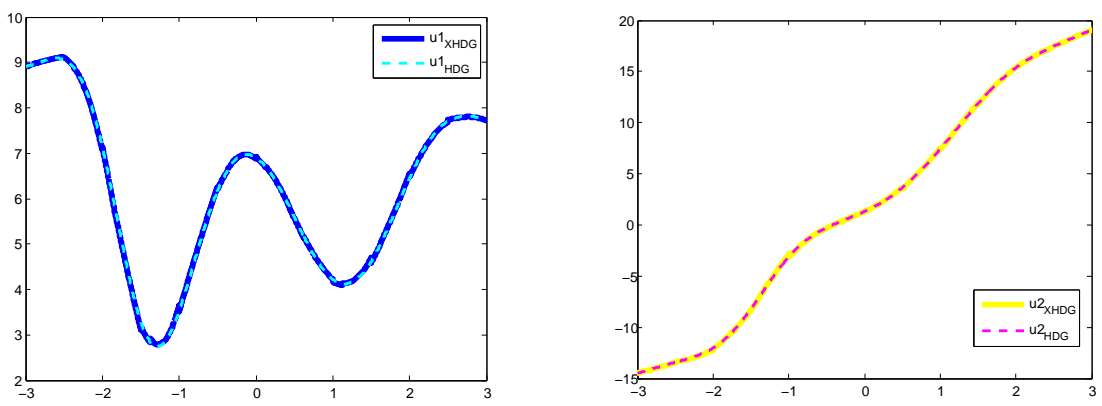

Fig. 15 Flow around several obstacles: $x$ component of the velocity along the line $x=1$ using HDG and X-HDG (left) y component of the velocity along the line $x=1$ using HDG and X-HDG (right).

uniform regular mesh (right), in both cases with degree $k=2$. As a result of this comparison, $\mathrm{X}-\mathrm{HDG}$ is observed to be in excellent agreement with the standard HDG reference solution, using a non-fitted mesh. To strengthen this argument, in Figure 15, x and y components of the velocity along the line $x=1$, obtained using HDG and X-HDG are compared. As expected, velocity solutions obtained using HDG and X-HDG are observed to be perfectly overlapping.

\section{Conclusions and final remarks}

We have derived an eXtended Hybridizable Discontinuous Galerkin (X-HDG) formulation for solving Stokes interface problems with unfitted meshes. The method combines the accuracy and efficiency of high-order hybridizable discontinuous Galerkin method with an XFEM philosophy that allows arbitrary material interfaces or boundaries that are not aligned with the computational mesh. In the proposed method, the interface is described by the zero contour of a level-set function based on high-order polynomials in the domain. Weak interface compatibility conditions are imposed in a consistent HDG setting for both Dirichlet and Neumann boundaries as well as for material interfaces, by introducing a new interfacial trace variable, similar to the one defined on the faces between elements. The interface trace variable is local to the elements and can be expressed as part of the solutions of the local solver, together with the element velocity, the velocity gradient, and the pressure. Thus, the method follows the code structure of usual HDG implementations and can be added as an extension of the local solver facilities. For bimaterial problems, Heaviside enrichments for the velocity, the velocity gradient, the pressure, and the trace velocity have been added to accommodate for two independent solution components inside the cut elements. As usual in HDG, the global linear system is only expressed in terms of the trace of the velocity on the mesh skeleton and the element-wise pressure average, only this time with more degrees of freedom over cut faces. The proposed work follows previous work on the Laplace problem $[14,15]$, with integration on cut elements and interfaces done by a high-order quadrature approach that was previously used for Laplace-type problems.

Six two-dimensional numerical examples have been presented demonstrating that the proposed method carries over the optimal convergence rates and accuracy of the HDG method to the unfitted mesh case, in the presence of Dirichlet voids, Neumann voids, and bimaterial interfaces. A test on a setting typical of two-phase flow demonstrates that the method is also applicable to settings with jumps in the pressure field, making it a promising 
approach for complex application problems that can profit from the accuracy and efficiency of HDG.

Acknowledgements This work was supported by the DAFOH2 project (Ministerio de Economia y Competitividad, MTM2013-46313-R), the Erasmus Mundus Joint Doctorate SEED project (European Comission, 2013-1436/001-001-EMJD) and the Catalan government (Generalitat de Catalunya, 2009SGR875). The authors also acknowledge Ms. Esther Sala-Lardies (Universitat Politècnica de Catalunya) for letting them use her library for numerical integration in cut elements.

\section{References}

1. Axelsson, O., Boyanova, P., Kronbichler, M., Neytcheva, M., Wu, X.: Numerical and computational efficiency of solvers for two-phase problems. Comput. Math. Appl. 65, 301-314 (2013)

2. Bastian, P.: A fully-coupled discontinuous Galerkin method for two-phase flow in porous media with discontinuous capillary pressure. Computational Geosciences 18(5), 779-796 (2014)

3. Burman, E., Hansbo, P.: Fictitious domain methods using cut elements: III. A stabilized Nitsche method for Stokes' problem. ESAIM: Mathematical Modelling and Numerical Analysis 48, 859-874 (2014)

4. Cheng, K.W., Fries, T.P.: Higher-order XFEM for curved strong and weak discontinuities. Int. J. Numer. Methods Eng. 82(5), 564-590 (2010)

5. Cockburn, B., Dong, B., Guzmán, J.: A superconvergent LDG-hybridizable Galerkin method for secondorder elliptic problems. Math. Comp. 77(264), 1887-1916 (2008)

6. Cockburn, B., Gopalakrishnan, J., Nguyen, N.C., Peraire, J., Sayas, F.J.: Analysis of HDG methods for Stokes flow. Math. Comp. 80(274), 723-760 (2011)

7. Cockburn, B., Nguyen, N.C., Peraire, J.: A comparison of HDG methods for Stokes flow. J. Sci. Comput. 45(1-3), 215-237 (2010)

8. Cockburn, B., Qiu, W., Shi, K.: Conditions for superconvergence of HDG methods for second-order elliptic problems. Math. Comp. 81(279), 1327-1353 (2012)

9. Dréau, K., Chevaugeon, N., Moës, N.: Studied X-FEM enrichment to handle material interfaces with higher order finite element. Comput. Methods Appl. Mech. Eng. 199(29-32), 1922-1936 (2010)

10. Fries, T., Omerović, S., Schöllhammer, D., Steidl, J.: Higher-order meshing of implicit geometriespart i: Integration and interpolation in cut elements. Computer Methods in Applied Mechanics and Engineering 313, 759 - 784 (2017). DOI https://doi.org/10.1016/j.cma.2016.10.019. URL http://www.sciencedirect.com/science/article/pii/S0045782516308696

11. Fries, T.P., Belytschko, T.: The extended/generalized finite element method: An overview of the method and its applications. International Journal for Numerical Methods in Engineering 84(3), 253-304 (2010). DOI 10.1002/nme.2904

12. Giorgiani, G., Fernández-Méndez, S., Huerta, A.: Hybridizable Discontinuous Galerkin with degree adaptivity for the incompressible Navier-Stokes equations. Comput. Fluids 98, 196-208 (2014)

13. Giorgiani, G., Modesto, D., Fernández-Méndez, S., Huerta, A.: High-order continuous and discontinuous Galerkin methods for wave problems. Int. J. Numer. Methods Fluids 73(10), 883-903 (2013)

14. Gürkan, C., Kronbichler, M. Fernández-Méndez, S.: eXtended hybridizable discontinuous Galerkin with heaviside enrichment for heat bimaterial problems. J. Sci. Comput. 72(2), 542-567 (2017)

15. Gürkan, C., Sala-Lardies, E., Kronbichler, M. Fernández-Méndez, S.: eXtended hybridizable discontinuous Galerkin (X-HDG) for void problems. J. Sci. Comput. 66(3), 1313-1333 (2016)

16. Hansbo, A., Hansbo, P.: An unfitted finite element method, based on Nitsche's method, for elliptic interface problems. Computer Methods in Applied Mechanics and Engineering 191(47), 5537-5552 (2002)

17. Hansbo, P., Larson, M., Zahedi, S.: A cut finite element method for a Stokes interface problem. Applied Numerical Mathematics 85, 90-114 (2014). DOI 10.1016/j.apnum.2014.06.009

18. Huynh, L.T., Nguyen, N., Peraire, J., Khoo, B.: A high-order hybridizable discontinuous Galerkin method for elliptic interface problems. Int. J. Numer. Meth. Eng. 93(2), 183-200 (2013)

19. Jacqmin, D.: Calculation of two-phase Navier-Stokes flows using phase-field modeling. Journal of Computational Physics 155(1), 96-127 (1999)

20. Kirby, R., Sherwin, S.J., Cockburn, B.: To CG or to HDG: A comparative study. J. Sci. Comput. 51(1), 183-212 (2011)

21. Matthias, K., Sven, G., Arnold, R.: Analysis of an XFEM discretization for stokes interface problems. SIAM Journal on Scientific Computing 38(2), A1019-A1043 (2016). DOI 10.1137/15M1011779

22. Mittal, R., Iaccarino, G.: Immersed boundary methods. Annual Review of Fluid Mechanics 37(1), 239261 (2005). DOI 10.1146/annurev.fluid.37.061903.175743 
23. Moës, N., Dolbow, J., Belytschko, T.: A finite element method for crack growth without remeshing. Int. J. Numer. Methods Eng. 46(1), 131-150 (1999)

24. Nguyen, N., Peraire, J., Cockburn, B.: An implicit high-order hybridizable discontinuous Galerkin method for the incompressible Navier-Stokes equations. Journal of Computational Physics 230(4), 1147-1170 (2011)

25. Paipuri, M., Fernández-Méndez, S., Tiago, C.: Comparison of high-order continuous and hybridizable discontinuous Galerkin methods in incompressible fluid flow problems. Mathematics and Computers in Simulation 153, 35-58 (2018). DOI https://doi.org/10.1016/j.matcom.2018.05.012

26. Pigeonneau, F., Saramito, P.: Discontinuous Galerkin finite element method applied to the coupled Navier-Stokes/Cahn-Hilliard equations. In: ICMF-2016 - 9th International Conference on Multiphase Flow. Florence, Italy (2016)

27. Wang, B., Khoo, B.: Hybridizable discontinuous Galerkin method (HDG) for Stokes interface flow. J. Comput. Phys. 247, 262-278 (2013)

28. Yakovlev, S., Moxey, D., Kirby, R.M., Sherwin, S.J.: To CG or to HDG: A comparative study in 3D. J. Sci. Comput. 67(1), 192-220 (2015) 